\title{
Infinite dimensional Ornstein-Uhlenbeck processes with unbounded diffusion - Approximation, quadratic variation, and Itô formula
}

\author{
John Karlsson and Jörg-Uwe Löbus \\ Matematiska institutionen \\ Linköpings universitet \\ SE-581 83 Linköping \\ Sverige
}

\begin{abstract}
The paper studies a class of Ornstein-Uhlenbeck processes on the classical Wiener space. These processes are associated with a diffusion type Dirichlet form whose corresponding diffusion operator is unbounded in the Cameron-Martin space. It is shown that the distributions of certain finite dimensional Ornstein-Uhlenbeck processes converge weakly to the distribution of such an infinite dimensional OrnsteinUhlenbeck process. For the infinite dimensional processes, the ordinary scalar quadratic variation is calculated. Moreover, relative to the stochastic calculus via regularization, the scalar as well as the tensor quadratic variation are derived. A related Itô formula is presented.
\end{abstract}

AMS subject classification (2010) primary 60J60 secondary 60G15

Keywords Infinite dimensional Ornstein-Uhlenbeck process, quadratic variation, Itô formula, weak approximation.

\section{Introduction}

Over the past two decades, infinite dimensional diffusion processes have become a central focus of stochastic analysis. One important class of infinite dimensional stochastic processes is the set of Ornstein-Uhlenbeck type processes.

We wish to investigate a fairly accessible representative of the relatively abstract class of infinite dimensional stochastic processes with unbounded diffusion introduced in [15, 24], 44, and [13]. Existence and representation of standard elements of the stochastic calculus such as quadratic variation and Itô formula may convince that these processes fit in the general concept of infinite dimensional stochastic processes. We would also like to emphasize that the just mentioned references deal with a class of stochastic processes taking values in certain path spaces, i.e. in Banach spaces.

As a result of this paper, we have scalar as well as tensor quadratic variation and the corresponding Itô formula available for a class of infinite dimensional Ornstein-Uhlenbeck processes with unbounded diffusion. For this we have used the recently developed stochastic calculus via regularization, see [22], [7, and [8]. Even if the diffusion of such infinite dimensional Ornstein-Uhlenbeck processes is governed by unbounded operators these processes can be weakly approximated by finite dimensional OrnsteinUhlenbeck processes.

Let $C_{0}\left([0,1] ; \mathbb{R}^{d}\right)$ denote the set of all continuous $\mathbb{R}^{d}$-valued functions $\gamma$ on $[0,1]$ with $\gamma(0)=0$ and let $C_{0}\left([0,1] ; \mathbb{R}^{d}\right)$ be endowed with the sup-norm. Introduce the following set of cylindrical functions over $\Omega \equiv C_{0}\left([0,1] ; \mathbb{R}^{d}\right)$

$$
\begin{aligned}
Y:=\{F(\gamma) & =f\left(\gamma\left(s_{1}\right), \ldots, \gamma\left(s_{k}\right)\right), \gamma \in \Omega: 0<s_{1}<\cdots<s_{k}=1, \\
& \left.s_{1}, \ldots, s_{k} \in\left\{\frac{l}{2^{n}}: l \in\left\{1, \ldots, 2^{n}\right\}\right\}, n \in \mathbb{N}, f \in C_{p}^{\infty}\left(\left(\mathbb{R}^{d}\right)^{k}\right), k \in \mathbb{N}\right\}
\end{aligned}
$$

where $C_{p}^{\infty}\left(\left(\mathbb{R}^{d}\right)^{k}\right)$ denotes the set of all infinitely differentiable real functions $f$ on $\left(\mathbb{R}^{d}\right)^{k}$ such that all its partial derivatives are of at most polynomial growth. For $F$ as in the above definition of $Y$ the 
gradient operator $D$ is given by

$$
D_{s} F(\gamma)=\sum_{i=1}^{k}\left(s_{i} \wedge s\right)\left(\nabla_{s_{i}} f\right)(\gamma), \quad s \in[0,1], \gamma \in \Omega,
$$

where $\left(\nabla_{s_{i}} f\right)(\gamma)=\left(\nabla_{s_{i}} f\right)\left(\gamma\left(s_{1}\right), \ldots, \gamma\left(s_{k}\right)\right)$ denotes the gradient of the function $f$ relative to the $i$ th variable while holding the other variables fixed. Let $\mathbb{H}$ denote the Cameron-Martin space, i.e. the space of all absolutely continuous $\mathbb{R}^{d}$-valued functions $f$ on $[0,1]$, with $f(0)=0$, equipped with inner product

$$
\langle\varphi, \psi\rangle_{\mathbb{H}}:=\int_{[0,1]}\left\langle\varphi^{\prime}(x), \psi^{\prime}(x)\right\rangle_{\mathbb{R}^{d}} d x
$$

Moreover let $S_{i}, i \in \mathbb{N}$, be the ONB in $\mathbb{H}$ consisting of the Schauder functions. Denote by $\mathcal{B}$ the $\sigma$-algebra of the Borel sets on $\Omega$. Let $\nu$ denote the Wiener measure on $(\Omega, \mathcal{B})$. Let $0<\lambda_{1} \leq \lambda_{2} \leq \ldots$ be a sequence of real numbers satisfying

$$
\sum_{m=0}^{\infty} 2^{-m} \lambda_{d 2^{m}}<\infty
$$

This paper is concerned with an Ornstein-Uhlenbeck type Dirichlet form $(\mathcal{E}, D(\mathcal{E}))$ obtained by the closure of the positive symmetric bilinear form

$$
\mathcal{E}(F, G)=\int \sum_{i=1}^{\infty} \lambda_{i}\left\langle D F, S_{i}\right\rangle_{\mathbb{H}}\left\langle D G, S_{i}\right\rangle_{\mathbb{H}} d \nu, \quad F, G \in Y,
$$

on $L^{2}(\nu)$. The Dirichlet form $(\mathcal{E}, D(\mathcal{E}))$ is a particular case of the set of Dirichlet forms associated with a certain class of infinite dimensional processes with unbounded diffusion introduced and studied in [15], 24], 4], and [13. In particular, it has been shown in [13, Proposition 4.2, that condition (1.1) is necessary and sufficient for closability of $(\mathcal{E}, Y)$ on $L^{2}(\nu)$. Furthermore, its closure $(\mathcal{E}, D(\mathcal{E}))$ on $L^{2}(\nu)$ is quasi-regular.

\subsection{Main results}

Let $G_{i}, i \in \mathbb{N}$, be a sequence of independent one-dimensional Ornstein-Uhlenbeck processes, i.e. we have $d G_{i}(t)=-G_{i}(t) d t+\sqrt{2} d W_{i}(t), t \geq 0$, for a sequence of independent one-dimensional Wiener processes $W_{i}, i \in \mathbb{N}$. This choice of $G_{i}$ corresponds to the fact that $G_{i}$ is related to the one-dimensional analogue of $\mathcal{E}$, namely $\mathcal{E}^{1}(f, g)=\int f^{\prime} g^{\prime} \varphi d x$ where $\varphi$ denotes the standard normal density. In the present paper we consider the Dirichlet form corresponding to (1.2) and show in Lemma 2.1 and Proposition 2.3 that the associated stochastic process has the representation

$$
X_{t}:=\sum_{i=1}^{\infty} G_{i}\left(\lambda_{i} t\right) \cdot S_{i}, \quad t \geq 0
$$

where the right-hand side converges in the norm of $C_{0}\left([0,1] ; \mathbb{R}^{d}\right)$ almost surely.

The main result of Section 2 is that, provided that

$$
\sum_{m=0}^{\infty} 2^{-\frac{m}{2}} \cdot \lambda_{d 2^{m}} \cdot\left(\max _{d 2^{m}+1<i \leq d 2^{m+1}}\left|G_{i}(0)\right|+m^{\frac{1}{2}}\right)<\infty,
$$

the sequence of processes $X_{t}^{(n)}:=\sum_{i=1}^{n} G_{i}\left(\lambda_{i} t\right) \cdot S_{i}, t \geq 0$, converges in distribution to $X_{t}, t \geq 0$, in the space $C_{C_{0}\left([0,1] ; \mathbb{R}^{d}\right)}([0, \infty))$ of all continuous $C_{0}\left([0,1] ; \mathbb{R}^{d}\right)$-valued trajectories.

In Section 3 we determine the scalar quadratic variation of $X$. For this let $L^{1}\left([0,1] ; \mathbb{R}^{d}\right)$ denote the set of all integrable $\mathbb{R}^{d}$-valued functions $\gamma$ on $[0,1]$ and let $L^{1}\left([0,1] ; \mathbb{R}^{d}\right)$ be endowed with the norm $\|\gamma\|_{1}:=$ $\sum_{i=1}^{d} \int_{0}^{1}\left|\gamma_{i}\right| d x$ where $\gamma=\left(\gamma_{1}, \ldots, \gamma_{d}\right)$. We consider $X$ as a process with either $C_{0}\left([0,1] ; \mathbb{R}^{d}\right)$-valued 
or $L^{1}\left([0,1] ; \mathbb{R}^{d}\right)$-valued trajectories. Correspondingly, let $\|\cdot\|$ denote the norm in either $C_{0}\left([0,1] ; \mathbb{R}^{d}\right)$ or $L^{1}\left([0,1] ; \mathbb{R}^{d}\right)$. Let $\xi_{i}, i \in \mathbb{N}$, be a sequence of independent identically distributed standard normal random variables and define

$$
\theta:=2 E\left[\left\|\sum_{i=1}^{\infty} \lambda_{i}^{\frac{1}{2}} \xi_{i} S_{i}\right\|^{2}\right]
$$

where we note that $\theta$ depends on the choice of the norm. Suppose now that

$$
\sum_{m=0}^{\infty} 2^{-\frac{m}{2}} \cdot \lambda_{d 2^{m+1}} \cdot\left(\max _{d 2^{m}+1<i \leq d 2^{m+1}}\left|G_{i}(0)\right|+m^{\frac{1}{2}}\right)<\infty
$$

and note the similarity to (1.4). Let $\tau^{n}=\left\{0=t_{0}^{n}, t_{1}^{n}, \ldots, t_{k(n)}^{n}=T\right\}, n \in \mathbb{N}$, be an arbitrary sequence of partitions on $[0, T], T>0$ such that $\lim _{n \rightarrow \infty}\left|\tau^{n}\right|=0$. We show that in the ucp sense (uniform convergence in probability)

$$
[X]_{t}:=\lim _{n \rightarrow \infty} \sum_{j: t_{j}^{n} \leq t}\left\|X_{t_{j}^{n}}-X_{t_{j-1}^{n}}\right\|^{2}=\theta t
$$

cf. Proposition 3.3. Furthermore in Proposition 3.5 we verify that for the scalar quadratic variation relative to the stochastic calculus via regularization, cf. [22], [7, and [8], it holds that

$$
\frac{1}{\delta} \int_{0}^{t}\left\|X_{s+\delta}-X_{s}\right\|^{2} d s \quad \underset{\delta \rightarrow 0}{\longrightarrow} \quad t \theta \quad \text { ucp on } t \in[0, \infty) .
$$

The aim of Section 4 is to determine the tensor quadratic variation of the process $X$ in the Banach space $L^{1}\left([0,1] ; \mathbb{R}^{d}\right) \widehat{\otimes}_{\pi} L^{1}\left([0,1] ; \mathbb{R}^{d}\right)$ relative to the stochastic calculus via regularization. That is, we examine

$$
[X]_{t}^{\otimes}:=\lim _{\delta \rightarrow 0} \int_{0}^{t} \frac{\left(X_{u+\delta}-X_{u}\right) \otimes\left(X_{u+\delta}-X_{u}\right)}{\delta} d u
$$

in the ucp sense with respect to the norm in $L^{1}\left([0,1] ; \mathbb{R}^{d}\right) \widehat{\otimes}_{\pi} L^{1}\left([0,1] ; \mathbb{R}^{d}\right)$. For this, let $\xi_{i}, i \in \mathbb{N}$, be a sequence of independent standard normal random variables and define

$$
\Theta:=2 E\left[\left(\sum_{i=1}^{\infty} \lambda_{i}^{\frac{1}{2}} \xi_{i} S_{i}\right) \otimes\left(\sum_{i^{\prime}=1}^{\infty} \lambda_{i^{\prime}}^{\frac{1}{2}} \xi_{i^{\prime}} S_{i^{\prime}}\right)\right] .
$$

Provided that we have (1.5) we prove

$$
[X]_{t}^{\otimes}=t \Theta, \quad t \in[0, \infty) .
$$

Section [5 is devoted to the Itô formula corresponding to (1.3). Using recent results presented in [7] and [8], our efforts in Sections 3 and 4 lead to

$$
\begin{aligned}
F\left(t, X_{t}\right)= & F\left(0, X_{0}\right)+\int_{0}^{t} \frac{\partial}{\partial s} F\left(s, X_{s}\right) d s+\int_{0}^{t}{ }_{B^{*}}\left\langle D F\left(s, X_{s}\right), d X_{s}\right\rangle_{B} \\
& +\frac{1}{2} \int_{0}^{t}\left(B \widehat{\otimes}_{\pi} B\right)^{*}\left\langle D^{2} F\left(s, X_{s}\right), \Theta\right\rangle_{\left(B \widehat{\otimes}_{\pi} B\right)^{* *}} d s
\end{aligned}
$$

where $B=L^{1}\left([0,1] ; \mathbb{R}^{d}\right)$. We also verify that, for a certain class of cylindrical functions $F$, the expression on the right hand side takes the well known form of the finite dimensional Itô formula.

We would like to emphasize that infinite dimensional Ornstein-Uhlenbeck processes of the form (1.3) allow a decomposition $X_{t}=Y_{t}+Z_{t}+A_{t}, t \geq 0$, where

$$
Y_{t}=\sum_{i=1}^{\infty} \int_{u=0}^{t} e^{-\lambda_{i} u} d W_{i}\left(e^{2 \lambda_{i} u}-1\right) \cdot S_{i},
$$


$Z_{t}=-\sum_{i=1}^{\infty} \int_{u=0}^{t} \lambda_{i} e^{-\lambda_{i} u} W_{i}\left(e^{2 \lambda_{i} u}-1\right) d u \cdot S_{i}$, and $A_{t}=\sum_{i=1}^{\infty} e^{-\lambda_{i} t} G_{i}(0) \cdot S_{i}$. This decomposition is reasonable since the process $Y$ has independent increments. Independent increments are particularly useful for calculating a quadratic variation. Furthermore under mild conditions, the parts $Z$ and $A$ have a zero quadratic variation. In addition, we observe that the components $G_{i}\left(\lambda_{i} t\right) \cdot S_{i}$ of $X_{t}=$ $\sum_{i=1}^{\infty} G_{i}\left(\lambda_{i} t\right) \cdot S_{i}$ are independent. In order to carry out the technical calculations of Sections 24 these independences are crucial.

Several times we use results from extreme value theory which in part we derive in the appendix.

\subsection{Some basic definitions}

Let $\Omega \equiv C_{0}\left([0,1] ; \mathbb{R}^{d}\right):=\left\{f \in C\left([0,1] ; \mathbb{R}^{d}\right): f(0)=0\right\}$ be the space of trajectories, endowed with the norm $\|f\|:=\sup \left\{\left|f_{i}(x)\right|: x \in[0,1], i \in\{1, \ldots, d\}\right\}$ where $f=\left(f_{1}, \ldots, f_{d}\right)$ and $f_{1}, \ldots, f_{d} \in C_{0}([0,1] ; \mathbb{R})$. Furthermore, let $L^{1}\left([0,1] ; \mathbb{R}^{d}\right)$ be endowed with the norm $\|f\|_{1}:=\sum_{i=1}^{d} \int_{0}^{1}\left|f_{i}\right| d x$ where $f=\left(f_{1}, \ldots, f_{d}\right)$ and $f_{1}, \ldots, f_{d} \in L^{1}([0,1] ; \mathbb{R})$. This choice of the norm in $L^{1}\left([0,1] ; \mathbb{R}^{d}\right)$ guarantees compatability with the tensor product in Section 4

Let $\left(e_{j}\right)_{j=1, \ldots, d}$ denote the standard basis in $\mathbb{R}^{d}$ and introduce the system of the Haar functions on $[0,1]$ by

$$
\begin{aligned}
& H_{1}(t)=1, \quad t \in[0,1], \\
& H_{2^{m}+k}(t)=\left\{\begin{array}{ll}
2^{\frac{m}{2}} & \text { if } t \in\left[\frac{2 k-2}{2^{m+1}}, \frac{2 k-1}{2^{m+1}}\right) \\
-2^{\frac{m}{2}} & \text { if } t \in\left[\frac{2 k-1}{2^{m+1}}, \frac{2 k}{2^{m+1}}\right) \\
0 & \text { otherwise }
\end{array} \quad k=1, \ldots, 2^{m}, m=0,1, \ldots .\right.
\end{aligned}
$$

Also define

$$
g_{d(r-1)+j}:=H_{r} \cdot e_{j}, \quad r \in \mathbb{N}, j \in\{1, \ldots, d\}
$$

and

$$
S_{i}(s):=\int_{0}^{s} g_{i}(u) d u, \quad \text { as well as }\left\langle S_{i}, \gamma\right\rangle:=\int_{0}^{1} g_{i}(u) d \gamma_{u}
$$

Introduce

$$
\tilde{Y}:=\left\{F(\gamma)=f\left(\left\langle S_{1}, \gamma\right\rangle, \ldots,\left\langle S_{k}, \gamma\right\rangle\right): f \in C_{p}^{\infty}\left(\mathbb{R}^{k}\right), k \in \mathbb{N}\right\}
$$

as well as

$$
\tilde{Y}_{n}:=\left\{F(\gamma)=f\left(\left\langle S_{1}, \gamma\right\rangle, \ldots,\left\langle S_{k}, \gamma\right\rangle\right): f \in C_{p}^{\infty}\left(\mathbb{R}^{k}\right), k \leq n\right\}, \quad n \in \mathbb{N}
$$

Lemma 1.1. We have $Y=\tilde{Y}$.

Proof. For the sake of clarity we concentrate on the case $d=1$. We note that for any dyadic point $s \in[0,1]$ we have $\gamma(s)=\int_{0}^{1} \mathbb{1}_{[0, s]}(u) d \gamma_{u}$. We also note that $\left\langle S_{i}, \gamma\right\rangle$ can be written as

$$
\begin{aligned}
\left\langle S_{i}, \gamma\right\rangle & =\int_{0}^{1} H_{i}(u) d \gamma_{u} \\
& =2 c \int_{0}^{1} \mathbb{1}_{\left[0, s_{2}\right]}(u) d \gamma_{u}-c \int \mathbb{1}_{\left[0, s_{1}\right]}(u) d \gamma_{u}-c \int \mathbb{1}_{\left[0, s_{3}\right]}(u) d \gamma_{u}
\end{aligned}
$$

where for $i=2^{m}+k, m=0,1, \ldots, k=1, \ldots, 2^{m}$, we have $c=2^{\frac{m}{2}}, s_{1}=(k-1) / 2^{m}, s_{2}=(2 k-1) / 2^{m+1}$, and $s_{3}=k / 2^{m}$. Thus, any element of $\tilde{Y}$ belongs to $Y$. As for the converse direction, we note that for any dyadic $s \in[0,1]$ it holds that

$$
\mathbb{1}_{[0, s]}(u)=\sum_{i=1}^{N_{s}} \alpha_{i} H_{i}(u)
$$

for some $N_{s} \in \mathbb{N}$ and $\alpha_{1}, \ldots, \alpha_{N_{s}} \in \mathbb{R}$. The statement follows. 


\section{Finite dimensional approximation of the infinite dimensional process}

In this section we turn our attention to finite dimensional Dirichlet forms $\left(\mathcal{E}_{n}, D\left(\mathcal{E}_{n}\right)\right), n \in \mathbb{N}$, on suitable finite dimensional subspaces of $L^{2}(\nu)$ given by the closure of

$$
\mathcal{E}_{n}(F, G)=\int \sum_{i=1}^{n} \lambda_{i}\left\langle D F, S_{i}\right\rangle_{\mathbb{H}}\left\langle D G, S_{i}\right\rangle_{\mathbb{H}} d \nu, \quad F, G \in \tilde{Y}_{n}
$$

where $0<\lambda_{1} \leq \lambda_{2} \leq \ldots$ is a sequence of constants. We assume that for a sequence of independent one-dimensional standard Wiener processes $W_{i}, i \in \mathbb{N}$, we have

$$
d G_{i}(t)=-G_{i}(t) d t+\sqrt{2} d W_{i}(t), \quad t \geq 0 .
$$

In particular, with a non-random initial value $G_{i}(0)$ the variable $G_{i}\left(\lambda_{i} t\right)$ is $N\left(G_{i}(0) e^{-\lambda_{i} t}, 1-e^{-2 \lambda_{i} t}\right)$ distributed, $t>0$. In other words the $G_{i}$ are independent Ornstein-Uhlenbeck processes on $\mathbb{R}$. Therefore we have

$$
d G_{i}\left(\lambda_{i} t\right)=-\lambda_{i} G_{i}\left(\lambda_{i} t\right) d t+\sqrt{2 \lambda_{i}} d V_{i}(t), \quad i \in \mathbb{N},
$$

where $V_{i}=1 / \sqrt{\lambda_{i}} W_{i}\left(\lambda_{i} t\right)$ is a Wiener process. Consequently $G_{i}\left(\lambda_{i} \cdot\right)$ is associated with the $L^{2}(\mathbb{R})$ generator $f \equiv f(x) \mapsto \lambda_{i} f^{\prime \prime}(x)-\lambda_{i} x f^{\prime}(x)$ and hence with the bilinear form $(f, g) \mapsto \lambda_{i} \int f^{\prime}(x) g^{\prime}(x) \varphi(x) d x$, $f, g \in C_{p}^{\infty}(\mathbb{R})$. Since $S_{i}, i \in \mathbb{N}$, is an orthonormal basis in the Cameron-Martin space and the $G_{i}$ s are independent, the stochastic process

$$
X_{t}^{(n)}=\sum_{i=1}^{n} G_{i}\left(\lambda_{i} t\right) \cdot S_{i}, \quad t \geq 0, n \in \mathbb{N}
$$

is associated with the bilinear form $\left(\varepsilon_{n}, D\left(\varepsilon_{n}\right)\right)$ given by (2.1). On the other hand, we recall from [13], Proposition 5.2, that $(\mathcal{E}, D(\mathcal{E}))$ given by the closure of

$$
\mathcal{E}(F, G)=\int \sum_{i=1}^{\infty} \lambda_{i}\left\langle D F, S_{i}\right\rangle_{\mathbb{H}}\left\langle D G, S_{i}\right\rangle_{\mathbb{H}} d \nu, \quad F, G \in \tilde{Y},
$$

is a quasi-regular Dirichlet form on $L^{2}(\nu)$ provided that $\sum_{m=0}^{\infty} 2^{-m} \cdot \lambda_{d 2^{m}}<\infty$. The objective of this section is to identify the stochastic process which is associated with $(\mathcal{E}, D(\mathcal{E}))$ and to approximate it by the sequence $X^{(n)}$. Lemma 2.1 - Proposition 2.3 are devoted to the well-definiteness and association with $(\mathcal{E}, D(\mathcal{E}))$ of the limit process.

Lemma 2.1. Assume that the processes $G_{i}\left(\lambda_{i} t\right), t \geq 0, i \in \mathbb{N}$, have been started with non-random initial values $G_{i}(0) \in \mathbb{R}, i \in \mathbb{N}$, such that $\sum_{i=1}^{\infty}\left|G_{i}(0)\right| S_{i}(s)$ converges in $C_{0}\left([0,1] ; \mathbb{R}^{d}\right)$. Let $X_{0}:=$ $\sum_{i=1}^{\infty} G_{i}(0) S_{i}(s)$. Then almost surely the sum $\sum_{i=1}^{\infty}\left|G_{i}\left(\lambda_{i} t\right)\right| \cdot S_{i}$, and hence

$$
X_{t}:=\sum_{i=1}^{\infty} G_{i}\left(\lambda_{i} t\right) \cdot S_{i}
$$

converges in $C_{0}\left([0,1] ; \mathbb{R}^{d}\right)$ uniformly for all $t \geq 0$.

Proof. We know that $G_{i}\left(\lambda_{i} t\right)$ is $N\left(G_{i}(0) e^{-\lambda_{i} t}, 1-e^{-2 \lambda_{i} t}\right)$-distributed and that for any standard normal random variable $\xi$ we have $P(\xi>x) \leq \frac{1}{\sqrt{2 \pi} x} e^{-\frac{x^{2}}{2}}, x>0$, see e.g. [18], Appendix II, Lemma 3.1. Thus it holds for all $i \in \mathbb{N}$, all $t \geq 0$, and all $y>\left|G_{i}(0)\right| e^{-\lambda_{i} t}$ that

$$
P\left(\left|G_{i}\left(\lambda_{i} t\right)\right|>y\right) \leq \frac{\sqrt{2}}{\sqrt{\pi}\left(y-\left|G_{i}(0)\right| e^{-\lambda_{i} t}\right)} \cdot \exp \left\{-\frac{1}{2}\left(y-\left|G_{i}(0)\right| e^{-\lambda_{i} t}\right)^{2}\right\} .
$$


Next we track down the numbering of the Schauder functions $S_{i}, i \in \mathbb{N}$. Let $i=d(r-1)+j$, $j \in\{1, \ldots, d\}$, and for $r \geq 2$, let $r=2^{m}+k$ where $m \in\{0,1, \ldots\}, k \in\left\{1, \ldots, 2^{m}\right\}$. Now set $n(i):=m$ if $i \geq 2 d+1$. We obtain

$$
\begin{aligned}
& \sum_{i=2 d+1}^{\infty} P\left(\left|G_{i}\left(\lambda_{i} t\right)\right|>\left|G_{i}(0)\right| e^{-\lambda_{i} t}+\sqrt{2 n(i)}\right) \leq \frac{1}{\sqrt{\pi}} \sum_{i=2 d+1}^{\infty} \frac{1}{\sqrt{n(i)}} e^{-n(i)} \\
& \quad=\frac{1}{\sqrt{\pi}} \sum_{m=1}^{\infty} d 2^{m} \frac{1}{\sqrt{m}} e^{-m}<\infty .
\end{aligned}
$$

The Borel-Cantelli lemma states now that $\nu$-a.s. there is an $i_{0}$ such that

$$
\left|G_{i}\left(\lambda_{i} t\right)\right| \leq\left|G_{i}(0)\right|+\sqrt{2 n(i)}, \quad i>i_{0} .
$$

Thus for $m$ such that $d 2^{m}+1>i_{0}$ we have

$$
\begin{aligned}
& \left|\sum_{i=d 2^{m}+1}^{\infty} G\left(\lambda_{i} t\right) S_{i}(s)\right| \leq \sum_{i=d 2^{m}+1}^{\infty}\left|G\left(\lambda_{i} t\right)\right| S_{i}(s) \leq \sum_{i=d 2^{m}+1}^{\infty}\left|G_{i}(0)\right| S_{i}(s)+\sum_{i=d 2^{m}+1}^{\infty} \sqrt{2 n(i)} S_{i}(s) \\
& \quad \leq \sum_{i=d 2^{m}+1}^{\infty}\left|G_{i}(0)\right| S_{i}(s)+\sum_{m^{\prime}=m}^{\infty} \sqrt{2 m^{\prime}} 2^{-\frac{m^{\prime}+2}{2}}, \quad s \in[0,1] .
\end{aligned}
$$

We have used the fact that for any $s \in[0,1]$ there is just one $d 2^{m^{\prime}}<i \leq d 2^{m^{\prime}+1}$ such that $S_{i}(s) \neq 0$. The last chain of inequalities implies the lemma.

We are now interested in the semigroup associated with the process (2.5). For a point $\mathbf{y} \equiv$ $\sum_{i=1}^{\infty} y_{i} S_{i} \in C_{0}\left([0,1] ; \mathbb{R}^{d}\right)$ with $y_{i} \in \mathbb{R}, i \in \mathbb{N}$, introduce

$$
T_{t} \mathbf{y}:=\sum_{i=1}^{\infty} y_{i} e^{-\lambda_{i} t} S_{i}
$$

and

$$
U_{t} \mathbf{y}:=\sum_{i=1}^{\infty} y_{i}\left(1-e^{-2 \lambda_{i} t}\right)^{\frac{1}{2}} S_{i}, \quad t>0
$$

Define $\mu_{t}:=\nu \circ U_{t}^{-1}, t>0$. Let $C_{b}\left(C_{0}\left([0,1] ; \mathbb{R}^{d}\right)\right)$ denote the space of all bounded continuous functions on $C_{0}\left([0,1] ; \mathbb{R}^{d}\right)$.

Proposition 2.2. (Mehler semigroup representation) Let $X_{0}:=\sum_{i=1}^{\infty} G_{i}(0) S_{i}$ be non-random such that $\sum_{i=1}^{\infty}\left|G_{i}(0)\right| S_{i}$ converges in $C_{0}\left([0,1] ; \mathbb{R}^{d}\right)$, cf. Lemma 2.1. Furthermore let $X_{t}$ be given by (2.5) and consider an arbitrary $F \in C_{b}\left(C_{0}\left([0,1] ; \mathbb{R}^{d}\right)\right) \cup \tilde{Y}$. Under these conditions we have

$$
E_{X_{0}}\left[F\left(X_{t}\right)\right]=\int F\left(T_{t} X_{0}+\mathbf{y}\right) \mu_{t}(d \mathbf{y}), \quad t>0 .
$$

Proof. It is sufficient to prove the claim for all cylindrical functions $F \in \tilde{Y}$ since $C_{b}\left(C_{0}\left([0,1] ; \mathbb{R}^{d}\right)\right) \cap \tilde{Y}$ is dense in $C_{b}\left(C_{0}\left([0,1] ; \mathbb{R}^{d}\right)\right)$ with respect to the $L^{1}(\nu)$-norm by the following reasoning. According to the approximation property as presented in 1] Theorem I.5.7 the simple cylindrical functions are dense in the set of all simple functions on $C_{0}\left([0,1] ; \mathbb{R}^{d}\right)$ with respect to the $L^{1}(\nu)$-norm. Noting that the simple functions on $C_{0}\left([0,1] ; \mathbb{R}^{d}\right)$ are dense in $L^{1}(\nu)$ and simple cylindrical function are dense in $\tilde{Y}$ with respect to the $L^{1}(\nu)$-norm we may conclude that $C_{b}\left(C_{0}\left([0,1] ; \mathbb{R}^{d}\right)\right) \cap \tilde{Y}$ is dense in $C_{b}\left(C_{0}\left([0,1] ; \mathbb{R}^{d}\right)\right)$.

For $i \in \mathbb{N}$ and $t>0$ let

$$
\psi_{t, G_{i}(0)}^{(i)}(x), x \in \mathbb{R}, \quad \text { be the density of } \quad N\left(G_{i}(0) e^{-\lambda_{i} t}, 1-e^{-2 \lambda_{i} t}\right)
$$


and note that $\psi_{t, G_{i}(0)}^{(i)}$ is the density of the process $G_{i}\left(\lambda_{i} t\right)$ at time $t>0$ when started in $G_{i}(0)$. We have

$$
\begin{aligned}
E_{X_{0}}\left[F\left(X_{t}\right)\right] & =E_{X_{0}}\left[F\left(\sum_{i=1}^{\infty} G_{i}\left(\lambda_{i} t\right) S_{i}\right)\right] \\
& =E_{X_{0}}\left[f\left(G_{1}\left(\lambda_{1} t\right), \ldots, G_{k}\left(\lambda_{k} t\right)\right)\right] \\
& =\int \ldots \int f\left(y_{1}, \ldots, y_{k}\right) \psi_{t, G_{1}(0)}^{(1)}\left(y_{1}\right) \ldots \psi_{t, G_{k}(0)}^{(k)}\left(y_{k}\right) d y_{1} \ldots d y_{k} \\
& =\int \ldots \int f\left(y_{1}+G_{1}(0) e^{-\lambda_{1} t}, \ldots, y_{k}+G_{k}(0) e^{-\lambda_{k} t}\right) \psi_{t, 0}^{(1)}\left(y_{1}\right) \ldots \psi_{t, 0}^{(k)}\left(y_{k}\right) d y_{1} \ldots d y_{k} \\
& =\int F\left(T_{t} X_{0}+\mathbf{y}\right) \mu_{t}(d \mathbf{y}) .
\end{aligned}
$$

Proposition 2.3. Suppose $\sum_{m=0}^{\infty} 2^{-m} \cdot \lambda_{d 2^{m}}<\infty$. Then the process (2.5) is associated with the Dirichlet form $(\mathcal{E}, D(\mathcal{E}))$ in the sense of

$$
\lim _{t \rightarrow 0} \int \frac{F-E \cdot\left[F\left(X_{t}\right)\right]}{t} \cdot H d \nu=\mathcal{E}(F, H), \quad F, H \in \tilde{Y} .
$$

Proof. Let $\varphi$ denote the density of $N(0,1)$. Using the notation of the proof of Proposition 2.2 and $H(\gamma)=h\left(\left\langle S_{1}, \gamma\right\rangle, \ldots,\left\langle S_{k}, \gamma\right\rangle\right)$ all we have to verify turns out to be a well-studied calculation in finite dimension,

$$
\begin{aligned}
\lim _{t \rightarrow 0} \int & \frac{F-E \cdot\left[F\left(X_{t}\right)\right]}{t} \cdot H d \nu \\
= & \lim _{t \rightarrow 0} \frac{1}{t} \int\left(\int f\left(x_{1}, \ldots, x_{k}\right)-f\left(y_{1}+x_{1} e^{-\lambda_{1} t}, \ldots, y_{k}+x_{k} e^{-\lambda_{k} t}\right) \times\right. \\
& \left.\times \psi_{t, 0}^{(1)}\left(y_{1}\right) \ldots \psi_{t, 0}^{(k)}\left(y_{k}\right) d y_{1}, \ldots d y_{k}\right) \cdot h\left(x_{1}, \ldots, x_{k}\right) \varphi\left(x_{1}\right) \ldots \varphi\left(x_{k}\right) d x_{1}, \ldots d x_{k} \\
= & \int \sum_{i=1}^{k} \lambda_{i}\left\langle D F, S_{i}\right\rangle_{\mathbb{H}}\left\langle D H, S_{i}\right\rangle_{\mathbb{H}} d \nu=\mathcal{E}(F, H) .
\end{aligned}
$$

We continue with the first step toward the approximation of the infinite dimensional process (2.5) by finite dimensional processes of the form (2.3).

Proposition 2.4. Let $\left(\mathcal{E}_{n}, D\left(\mathcal{E}_{n}\right)\right), n \in \mathbb{N}$, be given by (2.1) and let $X^{(n)}$ denote the associated process, see (2.3). Suppose

$$
\sum_{m=0}^{\infty} 2^{-\frac{m}{2}} \cdot \lambda_{d 2^{m}} \cdot m^{\frac{1}{2}}<\infty
$$

and choose an initial value $X^{(n)}(0):=\sum_{i=1}^{\infty} G_{i}(0) S_{i}(s)$ such that

$$
\sum_{m=0}^{\infty} 2^{-\frac{m}{2}} \max _{d 2^{m}+1<i \leq d 2^{m+1}}\left|G_{i}(0)\right| \cdot \lambda_{d 2^{m}}<\infty .
$$

Let $P_{n}, n \in \mathbb{N}$, denote the distribution of the process $X^{(n)}$ on the space $C_{C_{0}([0,1] ;: \mathbb{R} d)}([0, \infty))$ of continuous $C_{0}\left([0,1] ; \mathbb{R}^{d}\right)$-valued trajectories on $t \in[0, \infty)$. Then the family $P_{n}, n \in \mathbb{N}$, is relatively compact in the space of all probability measures on $C_{C_{0}\left([0,1] ; \mathbb{R}^{d}\right)}([0, \infty))$, endowed with the Prokhorov metric. 
Proof. Let $t \geq 0$ and $u \geq 0$. Conditioning on $\mathcal{F}_{t}:=\sigma\left(X_{s}: s \leq t\right)$ and taking into account the Markov property, the random variable

$$
Y_{i}:=G_{i}\left(\lambda_{i}(t+u)\right)-G_{i}\left(\lambda_{i} t\right)
$$

follows a $N\left(\mu_{i}, \sigma_{i}^{2}\right)$-distribution where

$$
\mu_{i}=G_{i}\left(\lambda_{i} t\right) \cdot\left(e^{-\lambda_{i} u}-1\right) \text { and } \sigma_{i}^{2}=1-e^{-2 \lambda_{i} u} .
$$

We aim to prove the claim by a conclusion similar to, for example, the final one in [16, Theorem 3.1.1. We have

$$
E\left[\left\|X_{t+u}^{(n)}-X_{t}^{(n)}\right\|^{4}\right]=E\left[\left(\sup _{s \in[0,1]}\left|\sum_{i=1}^{n} Y_{i} \cdot S_{i}(s)\right|\right)^{4}\right] .
$$

where the norm $\|\cdot\|$ is taken in $C_{0}\left([0,1] ; \mathbb{R}^{d}\right)$. It follows that

$$
\begin{aligned}
\left(\sup _{s \in[0,1]}\left|\sum_{i=1}^{n} Y_{i} \cdot S_{i}(s)\right|\right)^{4} & \leq\left(\left|Y_{1}\right|+\sup _{s \in[0,1]}\left|\sum_{m=0}^{\infty} \max _{d 2^{m}<i \leq d 2^{m+1}} Y_{i} \cdot S_{i}(s)\right|\right)^{4} \\
& \leq 8 Y_{1}^{4}+8\left(\sum_{m=0}^{\infty} 2^{-\frac{m+2}{2}} \max _{d 2^{m}<i \leq d 2^{m+1}}\left|Y_{i}\right|\right)^{4} .
\end{aligned}
$$

We have

$E\left[\left(\sum_{m=0}^{\infty} 2^{-\frac{m+2}{2}} \max _{d 2^{m}<i \leq d 2^{m+1}}\left|Y_{i}\right|\right)^{4}\right]=\sum_{m_{1}, \ldots, m_{4}=0}^{\infty} \prod_{j=1}^{4}\left(E\left[2^{-\frac{a_{j}\left(m_{j}+2\right)}{2}} \cdot \max _{d 2_{j}^{m}<i \leq d 2^{m_{j}+1}}\left|Y_{i}\right|^{a_{j}}\right]\right)^{\frac{1}{a_{j}}}$

where $a_{j} \equiv a_{j}\left(m_{1}, m_{2}, m_{3}, m_{4}\right)$ is the number of times $m_{j}$ appears among $m_{1}, m_{2}, m_{3}, m_{4}$. Now decompose $Y_{i}$ in the following manner. Let $\xi_{i} \sigma_{i}+\mu_{i}=Y_{i}, k \in\{1, \ldots, 4\}$, where $\xi_{i}$ is a standard normal random variable and $\mu_{i}$ and $\sigma_{i}$ are given by (2.9). Taking the conditional expectation gives

$$
E\left[2^{-\frac{k(m+2)}{2}} \cdot\left(\max _{d 2^{m}<i \leq d 2^{m+1}}\left|Y_{i}\right|\right)^{k}\right]=E\left[2^{-\frac{k(m+2)}{2}} \cdot E\left[\max _{d 2^{m}<i \leq d 2^{m+1}}\left|\xi_{i} \sigma_{i}+\mu_{i}\right|^{k} \mid \mathcal{F}_{t}\right]\right] .
$$

We recall that both, $\mu_{i}, i \in \mathbb{N}$, and $\xi_{i}, i \in \mathbb{N}$, are by construction sequences of independent random variables. Moreover, $\sigma_{i}>0$ is non-random, $\mu_{i}$ is measurable with respect to $\mathcal{F}_{t}$, and $\xi_{i}$ is independent of $\mathcal{F}_{t}$. It follows that

$$
\begin{aligned}
& E\left[2^{-\frac{k(m+2)}{2}} \cdot \max _{d 2^{m}<i \leq d 2^{m+1}}\left|Y_{i}\right|^{k}\right] \\
& \quad \leq 8 \cdot 2^{-\frac{k(m+2)}{2}} \cdot \max _{d 2^{m}<i \leq d 2^{m+1}} \sigma_{i}^{k} \cdot E\left[\max _{d 2^{m}<i \leq d 2^{m+1}}\left|\xi_{i}\right|^{k}\right]+8 E\left[2^{-\frac{k(m+2)}{2}} \cdot \max _{2^{m}<i \leq 2^{m+1}}\left|\mu_{i}\right|^{k}\right],
\end{aligned}
$$

$k \in\{1, \ldots, 4\}$. Now we apply $E\left[\max _{1 \leq i \leq n}\left|\xi_{i}\right|^{k}\right] \leq D(\ln n)^{\frac{k}{2}}$ for some $D>0$ independent of $n \geq 2$ which is a standard result from extreme value theory. We obtain

$$
\begin{aligned}
& E\left[2^{-\frac{k(m+2)}{2}} \cdot \max _{d 2^{m}<i \leq d 2^{m+1}}\left|Y_{i}\right|^{k}\right] \\
& \quad \leq 8 D_{1} \cdot 2^{-\frac{k(m+2)}{2}} \cdot \max _{d 2^{m}<i \leq d 2^{m+1}} \sigma_{i}^{k} \cdot m^{\frac{k}{2}}+8 E\left[2^{-\frac{k(m+2)}{2}} \cdot \max _{2^{m}<i \leq 2^{m+1}}\left|\mu_{i}\right|^{k}\right],
\end{aligned}
$$

$k \in\{1, \ldots, 4\}$, for some $D_{1}>0$ independent of $m \geq 1$. Next we recall that

$$
\mu_{i} \text { is } N\left(G_{i}(0) e^{-\lambda_{i} t}\left(1-e^{-\lambda_{i} u}\right),\left(1-e^{-2 \lambda_{i} t}\right)\left(1-e^{-\lambda_{i} u}\right)^{2}\right) \text {-distributed }
$$


and that $\sigma_{i}=\left(1-e^{-2 \lambda_{i} u}\right)^{\frac{1}{2}}$. Therefore we may write

$$
\mu_{i}=G_{i}(0) e^{-\lambda_{i} t}\left(1-e^{-\lambda_{i} u}\right)+\left(1-e^{-2 \lambda_{i} t}\right)^{\frac{1}{2}}\left(1-e^{-\lambda_{i} u}\right) \cdot \eta_{i}
$$

where $\eta_{i}, i \in \mathbb{N}$, is a sequence of independent $N(0,1)$-distributed random variables. Applying this to (2.13) we obtain

$$
\begin{aligned}
E & {\left[2^{-\frac{k(m+2)}{2}} \max _{d 2^{m}<i \leq d 2^{m+1}}\left|Y_{i}\right|^{k}\right] } \\
\leq & 8 D_{1} \cdot 2^{-\frac{k(m+2)}{2}} \cdot \max _{d 2^{m}<i \leq d 2^{m+1}} \sigma_{i}^{k} \cdot m^{\frac{k}{2}} \\
& +8 E\left[2^{-\frac{k(m+2)}{2}} \cdot \max _{d 2^{m}<i \leq d 2^{m+1}}\left|G_{i}(0) e^{-\lambda_{i} t}\left(1-e^{-\lambda_{i} u}\right)+\left(1-e^{-2 \lambda_{i} t}\right)^{\frac{1}{2}}\left(1-e^{-\lambda_{i} u}\right) \cdot \eta_{i}\right|^{k}\right] \\
& \leq 8 D_{1} \cdot 2^{-\frac{k(m+2)}{2}} \cdot\left(1-\exp \left\{-2 \lambda_{d 2^{m}} \cdot u\right\}\right)^{\frac{k}{2}} \cdot m^{\frac{k}{2}} \\
& +8^{2} \cdot 2^{-\frac{k(m+2)}{2}} \cdot\left(1-\exp \left\{-\lambda_{d 2^{m}} \cdot u\right\}\right)^{k}\left(\max _{d 2^{m}<i \leq d 2^{m+1}}\left|G_{i}(0)\right|^{k}+D_{1} \cdot m^{\frac{k}{2}}\right) \\
\leq & D_{2} \cdot 2^{-\frac{k(m+2)}{2}} \cdot \lambda_{d 2^{m}}^{k} \cdot u^{\frac{k}{2}} \cdot\left(\max _{d 2^{m}<i \leq d 2^{m+1}}\left|G_{i}(0)\right|^{k}+m^{\frac{k}{2}}\right), \quad u \in[0,1],
\end{aligned}
$$

$k \in\{1, \ldots, 4\}$, for some $D_{2}>0$ independent of $m \geq 1$ and $t \geq 0$. Taking into account conditions (2.7) and (2.8) we apply (2.14) to (2.12) and thus also to (2.10) and obtain

$$
E\left[\left\|X_{t+u}^{(n)}-X_{t}^{(n)}\right\|^{4}\right] \leq 8 E\left[Y_{1}^{4}\right]+8 E\left[\left(\sum_{m=0}^{\infty} 2^{-\frac{m+2}{2}} \max _{d 2^{m}<i \leq d 2^{m+1}}\left|Y_{i}\right|\right)^{4}\right] \leq C \cdot u^{2}, \quad u \in[0,1]
$$

for some $C>0$ independent of $n \in \mathbb{N}$ and $t \geq 0$. It follows now from a straight forward generalization of [2], Theorem 8.3, and [23], Corollary 2.1.4, to Banach space valued variables, that the family $P_{n}$, $n \in \mathbb{N}$, is relatively compact in the space $C_{C_{0}\left([0,1] ; \mathbb{R}^{d}\right)}([0, \infty))$ of continuous trajectories.

We now show convergence in distribution of $X_{t}^{(n)}=\sum_{i=1}^{n} G_{i}\left(\lambda_{i} t\right) \cdot S_{i}, t \geq 0$, to $X_{t}:=\sum_{i=1}^{\infty} G_{i}\left(\lambda_{i} t\right) \cdot S_{i}$ as $n \rightarrow \infty$.

Proposition 2.5. Under the assumptions of Proposition 2.4 the following holds.

(a) The process $X_{t}:=\sum_{i=1}^{\infty} G_{i}\left(\lambda_{i} t\right) \cdot S_{i}, t \geq 0$, has almost surely a continuous modification. We will use this modification from now on.

(b) Let $P_{n}$ and $P$ denote the distributions associated with the processes $X^{(n)}$ and $X$ respectively. The finite dimensional distributions of $X^{(n)}$ converge weakly to those of $X$. That is

$$
\begin{gathered}
\int \varphi\left(x_{1}, \ldots, x_{m}\right) d P_{n}\left(X^{(n)}\left(t_{1}\right) \in d x_{1}, \ldots, X^{(n)}\left(t_{m}\right) \in d x_{m}\right) \\
\underset{n \rightarrow \infty}{\longrightarrow} \int \varphi\left(x_{1}, \ldots, x_{m}\right) d P\left(X\left(t_{1}\right) \in d x_{1}, \ldots, X\left(t_{k}\right) \in d x_{m}\right)
\end{gathered}
$$

for all $m \in \mathbb{N}$ and all functions $\varphi$ belonging to some algebra of convergence determining functions over $\left(C_{0}\left([0,1] ; \mathbb{R}^{d}\right)\right)^{m}$, cf. [10] Section 4.4 .

Proof. (a) It follows from (2.10), (2.11), and (2.15) that

$$
E\left[\left\|X_{t+u}-X_{t}\right\|^{4}\right] \leq C \cdot u^{2}, \quad u \in[0,1]
$$

with the same $C>0$ as in (2.15). In fact, in (2.11) we turn to calculations which hold uniformly for all $n \in \mathbb{N}$ and also for estimating $E\left[\left\|X_{t+u}-X_{t}\right\|^{4}\right]$. By Kolmogorov's criterion, cf. [6], Theorem 3.3, we get continuity of the trajectories of $X$ as claimed. Alternatively, part(a) follows also from Proposition 3.3 below which is proved independently of the results of the present section. 
(b) By [10] Theorem 3.4.5 (b) it is sufficient to show that

$$
\int F_{1}\left(X^{(n)}\left(t_{1}\right)\right) \cdot \ldots \cdot F_{m}\left(X^{(n)}\left(t_{m}\right)\right) d P_{n} \quad \underset{n \rightarrow \infty}{ } \int F_{1}\left(X\left(t_{1}\right)\right) \cdot \ldots \cdot F_{m}\left(X\left(t_{m}\right)\right) d P
$$

for all $F_{i} \in \tilde{Y}$ of type $F_{i}(\mathbf{x})=f_{i 1}\left(\left\langle S_{1}, \mathbf{x}\right\rangle\right) \cdot \ldots \cdot f_{i k}\left(\left\langle S_{k}, \mathbf{x}\right\rangle\right), i=1, \ldots, m$. It is sufficient since the functions $\varphi\left(\mathbf{x}_{1}, \ldots, \mathbf{x}_{m}\right):=F_{1}\left(\mathbf{x}_{1}\right) \cdot \ldots \cdot F_{m}\left(\mathbf{x}_{m}\right)$ generate a convergence determining algebra over $\left(C_{0}\left([0,1] ; \mathbb{R}^{d}\right)\right)^{m}$. First we construct the Mehler representation relative to $X^{(n)}$. Recalling our approach and our notation in Proposition 2.2 we define for $\mathbf{y}^{(n)}=\sum_{i=1}^{n} y_{i} S_{i} \in C_{0}\left([0,1] ; \mathbb{R}^{d}\right)$ with $y_{i} \in \mathbb{R}, i \in \mathbb{N}$,

$$
T_{t}^{(n)} \mathbf{y}^{(n)}:=\sum_{i=1}^{n} y_{i} e^{-\lambda_{i} t} S_{i} \quad \text { and } \quad U_{t}^{(n)} \mathbf{y}^{(n)}:=\sum_{i=1}^{n} y_{i}\left(1-e^{-2 \lambda_{i} t}\right)^{\frac{1}{2}} S_{i}, \quad t \geq 0 .
$$

Furthermore, let

$$
\mu_{t}^{(n)}:=\nu^{(n)} \circ\left(U_{t}^{(n)}\right)^{-1}
$$

where $\nu^{(n)}$ is the projection of the Wiener measure to the linear span of $\left\{S_{1}, \ldots, S_{n}\right\}$. Let $\mathcal{T}_{t}^{(n)}$ denote the semi-group associated with $\left(\mathcal{E}_{n}, D\left(\mathcal{E}_{n}\right)\right)$ and let and $X_{0_{\tilde{Y}}}^{(n)}$ denote the non-random initial value of $X^{(n)}, n \in \mathbb{N}$. Let us consider cylindrical functions $F \in \tilde{Y}$ and $F_{n} \in \tilde{Y}, n \in \mathbb{N}$, of type $F(\mathbf{x})=$ $f_{1}\left(\left\langle S_{1}, \mathbf{x}\right\rangle\right) \cdot \ldots \cdot f_{k}\left(\left\langle S_{k}, \mathbf{x}\right\rangle\right)$ and $F_{n}(\mathbf{x})=f_{1}\left(\left\langle S_{1}, \mathbf{x}\right\rangle\right) \cdot \ldots \cdot f_{n \wedge k}\left(\left\langle S_{n \wedge k}, \mathbf{x}\right\rangle\right)$, for $\mathbf{x} \in C_{0}\left([0,1] ; \mathbb{R}^{d}\right)$. Following the proof of Proposition 2.2, the Mehler representation of $\mathcal{T}_{t}^{(n)} F_{n}$ is

$$
\left(\mathcal{T}_{t}^{(n)} F_{n}\right)\left(X_{0}^{(n)}\right)=\int F_{n}\left(T_{t}^{(n)} X_{0}^{(n)}+\mathbf{y}^{(n)}\right) \mu_{t}^{(n)}\left(d \mathbf{y}^{(n)}\right)
$$

where we mention that $\left(\mathcal{T}_{t}^{(n)} F_{n}\right)\left(X_{0}^{(n)}\right)$ is of the form $g_{1}\left(\left\langle S_{1}, \mathbf{x}\right\rangle\right) \cdot \ldots \cdot g_{n \wedge k}\left(\left\langle S_{n \wedge k}, \mathbf{x}\right\rangle\right)$. Using the fact that $F\left(T_{t}^{(n)} X_{0}^{(n)}+\mathbf{y}^{(n)}\right)=F\left(T_{t} X_{0}+\mathbf{y}\right)$ for $n \geq k$ we obtain

$$
\int F\left(T_{t}^{(n)} X_{0}^{(n)}+\mathbf{y}^{(n)}\right) \mu_{t}^{(n)}\left(d \mathbf{y}^{(n)}\right)=\int F\left(T_{t} X_{0}+\mathbf{y}\right) \mu_{t}(d \mathbf{y}), \quad n \geq k .
$$

Denoting by $\mathcal{T}_{t}, t \geq 0$, the semigroup associated with $\left(\mathcal{E}, D(\mathcal{E})\right.$ ), by (2.17) it follows that $\mathcal{T}_{t}^{(n)} F=\mathcal{T}_{t} F$, $t \geq 0$, for sufficiently large $n \in \mathbb{N}$. Let us fix $F_{1}, \ldots, F_{m} \in \tilde{Y}, m \in \mathbb{N}$. For sufficiently large $n \in \mathbb{N}$ it holds that

$$
\begin{aligned}
\int & F_{1}\left(X^{(n)}\left(t_{1}\right)\right) \cdot \ldots \cdot F_{m}\left(X^{(n)}\left(t_{m}\right)\right) d P_{n} \\
& =\int\left(\mathcal{T}_{t_{1}}^{(n)}\left(F_{1} \cdot \mathcal{T}_{t_{2}-t_{1}}^{(n)}\left(F_{2} \cdot \ldots \mathcal{T}_{t_{m}-t_{m-1}}^{(n)}\left(F_{m}\right) \ldots\right)\right)\right)\left(X_{0}^{(n)}\right) \nu^{(n)}\left(d X_{0}^{(n)}\right) \\
& =\int\left(\mathcal{T}_{t_{1}}\left(F_{1} \cdot \mathcal{T}_{t_{2}-t_{1}}\left(F_{2} \cdot \mathcal{T}_{t_{m}-t_{m-1}}\left(F_{m}\right) \ldots\right)\right)\right)\left(X_{0}\right) \nu\left(d X_{0}\right) \\
& =\int F_{1}\left(X\left(t_{1}\right)\right) \cdot \ldots \cdot F_{m}\left(X\left(t_{m}\right)\right) d P
\end{aligned}
$$

i.e. we have (2.16). The statement follows.

Corollary 2.6. Let $X^{(n)}, X, P_{n}$, and $P$ denote the processes and associated distributions introduced in (2.3) and (2.5). Under the hypotheses of Proposition 2.4 we have $P_{n} \rightarrow{ }_{n \rightarrow \infty} \quad P$ weakly on $C_{C_{0}\left([0,1] ; \mathbb{R}^{d}\right)}([0, \infty))$.

Proof. Combining the relative compactness result of Proposition 2.4 with the weak convergence of the finite dimensional distributions shown in Proposition 2.5 we prove the statement, see also Theorem 3.7.8 (b) of [10]. 


\section{Scalar quadratic variation}

We are now interested in a criterion for the increase of the sequence $\lambda_{i}, i \in \mathbb{N}$, in order to guarantee the finiteness of the quadratic variation. Subsequently we will use the concept of uniform convergence on compact subsets of $[0, \infty)$ in probability (abbreviated ucp), see e.g. [19] II.4 Definition p. 57.

Let $W_{i}, i \in \mathbb{N}$, be a sequence of independent one-dimensional standard Wiener processes and let $G_{i}(0) \in \mathbb{R}, i \in \mathbb{N}$, such that $\sum_{i=1}^{\infty}\left|G_{i}(0)\right| S_{i}$ converges in $C_{0}\left([0,1] ; \mathbb{R}^{d}\right)$. Then $G_{i}, i \in \mathbb{N}$, defined by

$$
\begin{aligned}
G_{i}(t) & :=e^{-t} G_{i}(0)+e^{-t} W_{i}\left(e^{2 t}-1\right) \\
& =e^{-t} G_{i}(0)+\int_{u=0}^{t} e^{-u} d W_{i}\left(e^{2 u}-1\right)+\int_{u=0}^{t} W_{i}\left(e^{2 u}-1\right) d e^{-u}, \quad t \geq 0,
\end{aligned}
$$

is a sequence of independent one-dimensional Ornstein-Uhlenbeck processes. Let the processes $X, Y$, and $Z$ on $t \in[0, \infty)$ be given by

$$
\begin{gathered}
X_{t}(s):=\sum_{i=1}^{\infty} G_{i}\left(\lambda_{i} t\right) \cdot S_{i}(s), \\
Y_{t}(s)=\sum_{i=1}^{\infty} \int_{u=0}^{t} e^{-\lambda_{i} u} d W_{i}\left(e^{2 \lambda_{i} u}-1\right) \cdot S_{i}(s),
\end{gathered}
$$

and

$$
Z_{t}(s)=-\sum_{i=1}^{\infty} \int_{u=0}^{t} \lambda_{i} e^{-\lambda_{i} u} W_{i}\left(e^{2 \lambda_{i} u}-1\right) d u \cdot S_{i}(s), \quad s \in[0,1] .
$$

provided that the infinite sums (3.1) and (3.2) converge almost surely in $C_{0}\left([0,1] ; \mathbb{R}^{d}\right)$. Recall also Lemma 2.1. In addition denote by $A_{t}:=\sum_{i=1}^{\infty} e^{-\lambda_{i} t} G_{i}(0) \cdot S_{i}, t \geq 0$, the non-random part of

$$
X_{t}=Y_{t}+Z_{t}+A_{t}, \quad t \geq 0
$$

We aim to analyze the quadratic variation of $X$. For this let $T>0$ be arbitrary and consider an arbitrary sequence of partitions of $[0, T]$,

$$
\tau^{n}=\left\{t_{0}^{n}, t_{1}^{n}, \ldots, t_{k(n)}^{n}: 0=t_{0}^{n} \leq t_{1}^{n} \leq \ldots \leq t_{k(n)}^{n}=T\right\}, \quad n \in \mathbb{N},
$$

with $\lim _{n \rightarrow \infty}\left|\tau^{n}\right|=0$. Here $|\cdot|$ denotes the mesh size, $|\tau|:=\max _{j}\left(t_{j}-t_{j-1}\right)$. Let $\|\cdot\|$ denote the norm in either $C_{0}\left([0,1] ; \mathbb{R}^{d}\right)$ or $L^{1}\left([0,1] ; \mathbb{R}^{d}\right)$. For $0 \leq t \leq T$ we introduce the ucp limit

$$
[X]_{t}:=\lim _{n \rightarrow \infty} \sum_{j: t_{j}^{n} \leq t}\left\|X_{t_{j}^{n}}-X_{t_{j-1}^{n}}\right\|^{2},
$$

where we note that this limit depends on the choice of the norm. In the same way we define

$$
[Y]_{t}:=\lim _{n \rightarrow \infty} \sum_{j: t_{j}^{n} \leq t}\left\|Y_{t_{j}^{n}}-Y_{t_{j-1}^{n}}\right\|^{2}
$$

and $[Z]_{t}:=\lim _{n \rightarrow \infty} \sum_{j: t_{j}^{n} \leq t}\left\|Z_{t_{j}^{n}}-Z_{t_{j-1}^{n}}\right\|^{2}$ provided that these limits exists ucp. For $t \in[0, T]$ introduce

$$
[A]_{t}:=\lim _{n \rightarrow \infty} \sum_{j: t_{j}^{n} \leq t}\left\|A_{t_{j}^{n}}-A_{t_{j-1}^{n}}\right\|^{2}
$$

whenever this limit exists uniformly in $t \in[0, T]$.

Let $\xi_{i}, i \in \mathbb{N}$, be a sequence of independent identically distributed standard normal random variables and define

$$
\theta:=2 E\left[\left\|\sum_{i=1}^{\infty} \lambda_{i}^{\frac{1}{2}} \xi_{i} S_{i}\right\|^{2}\right]
$$


Lemma 3.1. Suppose that

$$
\sum_{m=0}^{\infty} 2^{-\frac{m}{2}} \cdot \lambda_{d 2^{m+1}}^{\frac{1}{2}} \cdot m^{\frac{1}{2}}<\infty
$$

(a) The sum (3.1) converges almost surely in $C_{0}\left([0,1] ; \mathbb{R}^{d}\right)$. Furthermore, the limit (3.4) exists ucp on $t \in[0, \infty)$ for both norms, $C_{0}\left([0,1] ; \mathbb{R}^{d}\right)$ as well as $L^{1}\left([0,1] ; \mathbb{R}^{d}\right)$. It holds that $\theta<\infty$ and we have $[Y]_{t}=t \theta, t \in[0, \infty)$.

(b) For any $T>0$ we have for both norms, $C_{0}\left([0,1] ; \mathbb{R}^{d}\right)$ as well as $L^{1}\left([0,1] ; \mathbb{R}^{d}\right)$,

$$
\operatorname{Var}\left(\sum_{j: t_{j}^{n} \leq T}\left\|Y_{t_{j}^{n}}-Y_{t_{j-1}^{n}}\right\|^{2}\right) \leq C \sum_{j: t_{j}^{n} \leq T}\left(t_{j}^{n}-t_{j-1}^{n}\right)^{2}
$$

for some $C>0$ independent of $n \in \mathbb{N}$ and $\tau^{n}$.

Proof. Let $T>0$. For the sake of clarity, below we will use the abbreviations $t_{j}^{n} \equiv t_{j}$, and

$$
\Delta_{i, j} Y:=\int_{u=t_{j-1}}^{t_{j}} e^{-\lambda_{i} u} d W_{i}\left(e^{2 \lambda_{i} u}-1\right) .
$$

We observe that $\Delta_{i, j} Y$ is $N\left(0,2 \lambda_{i}\left(t_{j}-t_{j-1}\right)\right)$-distributed. Therefore

$$
E\left[\left\|\sum_{i=1}^{\infty}\left|\Delta_{i, j} Y\right| S_{i}\right\|^{4}\right] \leq d E\left[\left(\max _{0<i \leq d}\left|\Delta_{i, j} Y\right|+\sum_{m=0}^{\infty} 2^{-\frac{m+2}{2}} \max _{d 2^{m}<i \leq d 2^{m+1}}\left|\Delta_{i, j} Y\right|\right)^{4}\right] .
$$

In the case of the $C$-norm the last line is obvious since taking the sup-norm of $\sum_{i=1}^{\infty}\left|\Delta_{i, j} Y\right| S_{i}$ we just focus on one $s \in[0,1]$. We recall also that the $L^{1}$-norm of $S_{i}$ is bounded by the $C$-norm of $S_{i}$. Taking the fourth power and using the independence of the different Wiener processes it follows that we have to investigate terms of type

$$
E\left[\max _{d 2^{m}<i \leq d 2^{m+1}}\left|\Delta_{i, j} Y\right|^{k}\right], \quad k \in\{1, \ldots, 4\} .
$$

A known result from extreme value theory says that for a sequence $\xi_{i}, i \in \mathbb{N}$, of independent $N(0,1)$ distributed random variables we have $E\left[\max _{1 \leq i \leq r}\left|\xi_{i}\right|^{k}\right] \leq c_{k}(\ln r)^{\frac{k}{2}}$ for some $c_{k}>0$ independent of $r \geq 2$. For example see [9], Example 3.3.29, and [20, Proposition 2.1 (iii), where $a_{n}=c_{n}$ and $b_{n}=d_{n}$. Recalling that $\lambda_{i}$ is an increasing sequence it follows that

$$
E\left[\max _{d 2^{m}<i \leq d 2^{m+1}}\left|\Delta_{i, j} Y\right|^{k}\right] \leq d_{k} \lambda_{d 2^{m+1}}^{\frac{k}{2}} \cdot m^{\frac{k}{2}} \cdot\left(t_{j}-t_{j-1}\right)^{\frac{k}{2}},
$$

$k \in\{1, \ldots, 4\}$ for a suitable $d_{k}>0$ independent of $m$ and $\lambda_{i}, i \in \mathbb{N}$. Piecing everything together and keeping (3.5) in mind we obtain

$$
\begin{gathered}
E\left[\left(\max _{0<i \leq d}\left|\Delta_{i, j} Y\right|+\sum_{m=0}^{\infty} 2^{-\frac{m+2}{2}} \max _{d 2^{m}<i \leq d 2^{m+1}}\left|\Delta_{i, j} Y\right|\right)^{4}\right] \\
\leq C_{1} \cdot\left(\lambda_{d}^{\frac{1}{2}}+\sum_{m=0}^{\infty} 2^{-\frac{m}{2}} \cdot \lambda_{d 2^{m+1}}^{\frac{1}{2}} \cdot m^{\frac{1}{2}}\right)^{4} \cdot\left(t_{j}-t_{j-1}\right)^{2}
\end{gathered}
$$

for some $C_{1}>0$ independent of $\lambda_{i}, i \in \mathbb{N}, n \in \mathbb{N}$, and $\tau^{n}$. We get finiteness in (3.6) for any partition 
$\tau^{n}$. Thus $E\left[\left\|\sum_{i=1}^{\infty} \Delta_{i, j} Y S_{i}\right\|^{2}\right]$ is also finite. Applying Kolmogorov's inequality we obtain

$$
\begin{gathered}
P\left(\sup _{t \leq T} \mid \sum_{j: t_{j} \leq t}\left(\left\|\sum_{i=1}^{\infty} \Delta_{i, j} Y S_{i}\right\|^{2}-E\left[\left\|\sum_{i=1}^{\infty} \Delta_{i, j} Y S_{i}\right\|^{2}\right]\right) \geq \geq \varepsilon\right. \\
\leq \frac{1}{\varepsilon^{2}} \sum_{j} \operatorname{Var}\left(\left\|\sum_{i=1}^{\infty} \Delta_{i, j} Y S_{i}\right\|^{2}\right) \leq \frac{1}{\varepsilon^{2}} \sum_{j} E\left[\left\|\left|\sum_{i=1}^{\infty}\right| \Delta_{i, j} Y \mid S_{i}\right\|^{4}\right] \\
\leq \frac{d}{\varepsilon^{2}} \sum_{j} E\left[\left(\max _{0<i \leq d}\left|\Delta_{i, j} Y\right|+\sum_{m=0}^{\infty} 2^{-\frac{m+2}{2}} \max _{d 2^{m}<i \leq d 2^{m+1}}\left|\Delta_{i, j} Y\right|\right)^{4}\right] .
\end{gathered}
$$

We note that the finiteness of the right-hand side implies that the sum (3.1) converges almost surely in $C_{0}\left([0,1] ; \mathbb{R}^{d}\right)$. Continuing from (3.7) and using (3.6) we get

$$
\begin{aligned}
& P\left(\sup _{t \leq T}\left|\sum_{j: t_{j} \leq t}\left\|\sum_{i=1}^{\infty} \Delta_{i, j} Y S_{i}\right\|^{2}-E\left[\left\|\sum_{i=1}^{\infty} \Delta_{i, j} Y S_{i}\right\|^{2}\right]\right| \geq \varepsilon\right) \\
& \quad \leq \frac{d C_{1}}{\varepsilon^{2}} \cdot\left(\lambda_{d}^{\frac{1}{2}}+\sum_{m=0}^{\infty} 2^{-\frac{m}{2}} \cdot \lambda_{d 2^{m+1}}^{\frac{1}{2}} \cdot m^{\frac{1}{2}}\right)^{4} \sum_{j: t_{j} \leq t}\left(t_{j}-t_{j-1}\right)^{2} \underset{|\tau| \rightarrow 0}{\longrightarrow} 0 .
\end{aligned}
$$

It follows that for any sequence of partitions $\tau^{n}, n \in \mathbb{N}$ we have convergence ucp. We also note that

$$
\begin{aligned}
& \lim _{|\tau| \rightarrow 0} \sum_{j: t_{j} \leq t} E\left[\left\|\sum_{i=1}^{\infty} \Delta_{i, j} Y S_{i}\right\|^{2}\right] \\
& =\lim _{|\tau| \rightarrow 0} \sum_{j: t_{j} \leq t} E\left[2\left(t_{j}-t_{j-1}\right)\left\|\sum_{i=1}^{\infty} \frac{\lambda_{i}^{\frac{1}{2}} \Delta_{i, j} Y}{\left(\operatorname{Var}\left(\Delta_{i, j} Y\right)\right)^{\frac{1}{2}}} S_{i}\right\|^{2}\right] \\
& =t \theta .
\end{aligned}
$$

This shows (a). Part (b) follows from (3.7), (3.6), and (3.5).

Lemma 3.2. (a) Suppose that

$$
\sum_{m=0}^{\infty} 2^{-\frac{m}{2}} \max _{d 2^{m}+1<i \leq d 2^{m+1}}\left|G_{i}(0)\right| \cdot \lambda_{d 2^{m+1}}<\infty .
$$

Then $\sum_{i=1}^{\infty}\left|G_{i}(0)\right| S_{i}$ converges in $C_{0}\left([0,1] ; \mathbb{R}^{d}\right)$, i.e. $A_{t}=\sum_{i=1}^{\infty} e^{-\lambda_{i} t} G_{i}(0) \cdot S_{i}, t \geq 0$, is well-defined. Furthermore, the limit $[A]_{t}=\lim _{n \rightarrow \infty} \sum_{j: t_{j}^{n} \leq t}\left\|A_{t_{j}^{n}}-A_{t_{j-1}^{n}}\right\|^{2}$ exists uniformly in $t \in[0, T]$ for both norms, $C_{0}\left([0,1] ; \mathbb{R}^{d}\right)$ and $L^{1}\left([0,1] ; \mathbb{R}^{d}\right)$, and is constant zero on $[0, T]$.

(b) Suppose that

$$
\sum_{m=0}^{\infty} 2^{-\frac{m}{2}} \cdot \lambda_{d 2^{m+1}} \cdot m^{\frac{1}{2}}<\infty
$$

Under this condition the sum (3.2) converges almost surely in $C_{0}\left([0,1] ; \mathbb{R}^{d}\right)$. Moreover, it holds that the limit $[Z]_{t}=\lim _{n \rightarrow \infty} \sum_{j: t_{j}^{n} \leq t}\left\|Z_{t_{j}^{n}}-Z_{t_{j-1}^{n}}\right\|^{2}$ exists ucp on $t \in[0, \infty)$ for both norms, $C_{0}\left([0,1] ; \mathbb{R}^{d}\right)$ as well as $L^{1}\left([0,1] ; \mathbb{R}^{d}\right)$. Furthermore the limit is constant zero.

Proof. Similar as in the proof of the previous lemma, below we will use the abbreviations $t_{j}^{n} \equiv t_{j}$, and $\Delta_{i, j} A:=G_{i}(0) e^{-\lambda_{i} t_{j}}-G_{i}(0) e^{-\lambda_{i} t_{j-1}}$. 
(a) We obtain

$$
\begin{aligned}
& \sum_{j: t_{j} \leq T}\left\|\sum_{i=1}^{\infty} \Delta_{i, j} A S_{i}\right\|^{2} \\
& \leq \sum_{j: t_{j} \leq T}\left(\sum_{1 \leq i \leq d}\left|\Delta_{i, j} A\right|+\sum_{m=0}^{\infty} 2^{-\frac{m+2}{2}} \sum_{d 2^{m}<i \leq d 2^{m+1}}\left|\Delta_{i, j} A\right|\right)^{2} \\
& \leq \sum_{j:<t_{j} \leq T}\left(t_{j}-t_{j-1}\right)^{2}\left(d \cdot \max _{1 \leq i \leq d}\left|G_{i}(0)\right| \cdot \lambda_{d}+d \cdot \sum_{m=0}^{\infty} 2^{-\frac{m+2}{2}} \max _{d 2^{m}+1<i \leq d 2^{m+1}}\left|G_{i}(0)\right| \cdot \lambda_{d 2^{m+1}}\right)^{2} \\
& \underset{|\tau| \rightarrow 0}{\longrightarrow} 0
\end{aligned}
$$

where we have used $\left|\Delta_{i, j} A\right| \leq\left|G_{i}(0)\right| \lambda_{i} \cdot\left(t_{j}-t_{j-1}\right)$ and the assumption that $\lambda_{i}>0$ is non-decreasing in $i \in \mathbb{N}$. We get part (a) of the lemma.

(b) Set $\Delta_{i, j} Z:=-\int_{u=t_{j-1}}^{t_{j}} \lambda_{i} e^{-\lambda_{i} u} W_{i}\left(e^{2 \lambda_{i} u}-1\right) d u$. With $\xi_{i, j}:=\sup _{u \in\left[t_{j-1}, t_{j}\right]} e^{-\lambda_{i} u} \cdot W_{i}\left(e^{2 \lambda_{i} u}-1\right)$ we have

$$
\left|\Delta_{i, j} Z\right| \leq \xi_{i, j} \cdot \lambda_{i} \cdot\left(t_{j}-t_{j-1}\right)
$$

By Lemma A.4 we may find a constant $c>0$ independent of $j$ such that

$$
E\left[\max _{d 2^{m}<i \leq d 2^{m+1}} \xi_{i, j}^{k}\right] \leq c\left(\left(\ln \left(\lambda_{d 2^{m+1}}+1\right)\right)^{\frac{k}{2}}+m^{\frac{k}{2}}\right), \quad m \in\{0,1, \ldots\}, k=1,2,
$$

where for $\lambda_{d 2^{m+1}}<1$ we keep in mind the argumentation of Lemma 3.1. We follow the ideas of the proof of Lemma 3.1 (a) to obtain

$$
\begin{aligned}
& P\left(\sum_{j: t_{j} \leq T}\left\|\sum_{i=1}^{\infty} \Delta_{i, j} Z S_{i}\right\|^{2} \geq \varepsilon\right) \leq \frac{1}{\varepsilon} \sum_{j: t_{j} \leq T} E\left[\left\|\sum_{i=1}^{\infty} \Delta_{i, j} Z S_{i}\right\|^{2}\right] \\
& \quad \leq \frac{d}{\varepsilon} \sum_{j: t_{j} \leq T} E\left[\left(\max _{0<i \leq d}\left|\Delta_{i, j} Z\right|+\sum_{m=0}^{\infty} 2^{-\frac{m+2}{2}} \max _{d 2^{m}<i \leq d 2^{m+1}}\left|\Delta_{i, j} Z\right|\right)^{2}\right] \\
& \quad \leq \frac{d D_{1}}{\varepsilon} \sum_{j: t_{j} \leq T} E\left[\left(\max _{0<i \leq d} \xi_{i, j} \cdot \lambda_{d}+\sum_{m=0}^{\infty} 2^{-\frac{m+2}{2}} \max _{d 2^{m}<i \leq d 2^{m+1}} \xi_{i, j} \cdot \lambda_{d 2^{m+1}}\right)^{2}\right] \cdot\left(t_{j}-t_{j-1}\right)^{2} \\
& \quad \leq \frac{d D_{2}}{\varepsilon}\left(\lambda_{d}+\sum_{m=0}^{\infty} 2^{-\frac{m+2}{2}} \cdot \lambda_{d 2^{m+1}} \cdot m^{\frac{1}{2}}\right)^{2} \sum_{j: t_{j} \leq T}\left(t_{j}-t_{j-1}\right)^{2} \underset{|\tau| \rightarrow 0}{\longrightarrow} 0
\end{aligned}
$$

for some suitable constants $D_{1}>0$ and $D_{2}>0$ independent of $\lambda_{i}, i \in \mathbb{N}$, and $\tau^{n}$. En passant we have verified that the sum (3.2) converges almost surely in $C_{0}\left([0,1] ; \mathbb{R}^{d}\right)$. We have proved part (b) of the lemma.

Proposition 3.3. Assume (3.9) and (3.10). Then $[X]_{t}=[Y]_{t}=t \theta, t \in[0, \infty)$ for both norms, $C_{0}\left([0,1] ; \mathbb{R}^{d}\right)$ and $L^{1}\left([0,1] ; \mathbb{R}^{d}\right)$.

Proof. Below we use the previous abbreviations $\Delta_{i, j} Y, \Delta_{i, j} Z$, and $\Delta_{i, j} A$. Let $T>0$. We have for both 
norms, in $C_{0}\left([0,1] ; \mathbb{R}^{d}\right)$ and $L^{1}\left([0,1] ; \mathbb{R}^{d}\right)$,

$$
\begin{aligned}
& \left|\sum_{j: t_{j}<T}\left\|\sum_{i=1}^{\infty} G_{i}\left(\lambda_{i} t_{j}\right) \cdot S_{i}-\sum_{i=1}^{\infty} G_{i}\left(\lambda_{i} t_{j-1}\right) \cdot S_{i}\right\|^{2}-\sum_{j: t_{j}<T}\left\|\sum_{i=1}^{\infty} \Delta_{i, j} Y S_{i}\right\|^{2}\right| \\
& \leq 2 \sum_{j: t_{j}<T}\left\|\sum_{i=1}^{\infty} \Delta_{i, j} Y S_{i}\right\| \cdot\left\|\sum_{i^{\prime}=1}^{\infty}\left(\Delta_{i^{\prime}, j} Z S_{i^{\prime}}+\Delta_{i^{\prime}, j} A S_{i^{\prime}}\right)\right\| \\
& \quad+\sum_{j: t_{j}<T}\left\|\sum_{i=1}^{\infty}\left(\Delta_{i, j} Z S_{i}+\Delta_{i, j} A S_{i}\right)\right\|^{2} .
\end{aligned}
$$

We study the expectation of the sum of the absolute values of the mixed terms in (3.13) i.e.

$$
\mathbf{E}(\tau):=\sum_{j: t_{j}<T} E\left[\left\|\sum_{i=1}^{\infty} \Delta_{i, j} Y S_{i}\right\| \cdot\left\|\sum_{i^{\prime}=1}^{\infty}\left(\Delta_{i^{\prime}, j} Z S_{i^{\prime}}+\Delta_{i^{\prime}, j} A S_{i^{\prime}}\right)\right\|\right],
$$

where the partition $\tau$ is given by $\left(t_{j}\right)_{j=1}^{k}$. By the Schwarz inequality applied as in

$$
E\left[\left\|\sum_{i}\right\| \cdot\left\|\sum_{i^{\prime}}\right\|\right] \leq d^{2}\left(E\left[\left\|\sum_{i}\right\|_{C_{0}\left([0,1] ; \mathbb{R}^{d}\right)}^{2}\right]\right)^{\frac{1}{2}} \cdot\left(E\left[\left\|\sum_{i^{\prime}}\right\|_{C_{0}\left([0,1] ; \mathbb{R}^{d}\right)}^{2}\right]\right)^{\frac{1}{2}}
$$

and calculations similar to (3.6), (3.11), and (3.12) we verify

$$
\begin{aligned}
\mathbf{E}(\tau) & \leq D_{3}\left(\lambda_{d}^{\frac{1}{2}}+\sum_{m=0}^{\infty} 2^{-\frac{m}{2}} \cdot \lambda_{d 2^{m+1}}^{\frac{1}{2}} \cdot m^{\frac{1}{2}}\right) \times \\
& \times\left(\left(\max _{0<i \leq d}\left|G_{i}(0)\right|+1\right) \cdot \lambda_{d}+\sum_{m=0}^{\infty} 2^{-\frac{m}{2}}\left(\max _{d 2^{m}<i \leq d 2^{m+1}}\left|G_{i}(0)\right|+m^{\frac{1}{2}}\right) \cdot \lambda_{d 2^{m+1}}\right)^{2} \times \\
& \times\left(\sum_{j: t_{j}<T}\left(t_{j}-t_{j-1}\right)^{2}\right)^{\frac{1}{2}} \underset{|\tau| \rightarrow 0}{\longrightarrow} 0
\end{aligned}
$$

for a suitable constant $D_{3}>0$. We obtain

$$
P\left(\sum_{j: t_{j}<T}\left\|\sum_{i=1}^{\infty} \Delta_{i, j} Y S_{i}\right\| \cdot\left\|\sum_{i^{\prime}=1}^{\infty}\left(\Delta_{i^{\prime}, j} Z S_{i^{\prime}}+\Delta_{i^{\prime}, j} A S_{i^{\prime}}\right)\right\| \geq \varepsilon\right) \leq \frac{\mathbf{E}(\tau)}{\varepsilon} .
$$

This and (3.14) show that

$$
\sum_{j: t_{j}<t}\left\|\sum_{i=1}^{\infty} \Delta_{i, j} Y S_{i}\right\| \cdot\left\|\sum_{i^{\prime}=1}^{\infty}\left(\Delta_{i^{\prime}, j} Z S_{i^{\prime}}+\Delta_{i^{\prime}, j} A S_{i^{\prime}}\right)\right\| \underset{|\tau| \rightarrow 0}{\longrightarrow} 0
$$

ucp on $t \in[0, \infty)$. Similarly, applying Lemma 3.2 (a) and (b),

$$
\sum_{j: t_{j}<T}\left\|\sum_{i=1}^{\infty}\left(\Delta_{i, j} Z S_{i}+\Delta_{i, j} A S_{i}\right)\right\|^{2} \underset{|\tau| \rightarrow 0}{\longrightarrow} 0
$$

ucp on $t \in[0, \infty)$. Relations (3.15), (3.16), and (3.13) prove the statement.

For $r \in \mathbb{R}$ let $[r]$ denote the largest integer that does not exceed $r$. For $0 \leq a<\delta$ and $t>0$ define

$$
\langle Y\rangle_{a} \equiv\langle Y\rangle_{a ; t}(\delta):=\sum_{k=1}^{\left[\frac{t-a}{\delta}\right]+1}\left(\left\|Y_{a+k \delta}-Y_{a+(k-1) \delta}\right\|^{2}-E\left[\left\|Y_{a+k \delta}-Y_{a+(k-1) \delta}\right\|^{2}\right]\right)
$$

where as above $\|\cdot\|$ is the norm in either $C_{0}\left([0,1] ; \mathbb{R}^{d}\right)$ or $L^{1}\left([0,1] ; \mathbb{R}^{d}\right)$. 
Lemma 3.4. Suppose (3.5). We have

$$
\lim _{\delta \rightarrow 0} \sup _{0 \leq a<\delta} E\left[\left(\langle Y\rangle_{a ; t}(\delta)\right)^{2}\right]=0 .
$$

Proof. This is a particular case of Lemma 3.1 (b).

Proposition 3.5. Assume (3.9) and (3.10). Then we have

$$
\frac{1}{\delta} \int_{0}^{t}\left\|X_{s+\delta}-X_{s}\right\|^{2} d s \quad \underset{\delta \rightarrow 0}{\longrightarrow} \quad t \theta \quad \text { ucp on } t \in[0, \infty) .
$$

Proof. Let us use the same notation as in Lemma 3.4. We obtain for all $t \in[0, \infty)$ and $\varepsilon>0$

$$
\begin{aligned}
& P\left(\left|\int_{0}^{t}\left\|Y_{s+\delta}-Y_{s}\right\|^{2}-E\left[\left\|Y_{s+\delta}-Y_{s}\right\|^{2}\right] d s\right| \geq \delta \varepsilon\right) \\
& \quad=P\left(\left|\int_{0}^{\delta}\langle Y\rangle_{a} d a\right| \geq \delta \varepsilon\right) \leq \frac{1}{\varepsilon^{2} \delta^{2}} E\left[\left(\int_{0}^{\delta}\langle Y\rangle_{a} d a\right)^{2}\right] .
\end{aligned}
$$

Furthermore, we have

$$
E\left[\left(\int_{0}^{\delta}\langle Y\rangle_{a} d a\right)^{2}\right] \leq \delta \int_{0}^{\delta} E\left[\langle Y\rangle_{a}^{2}\right] d a \leq \delta^{2} \sup _{0 \leq a<\delta} E\left[\left(\langle Y\rangle_{a ; t}(\delta)\right)^{2}\right] .
$$

As in the proof of Lemma 3.1 (a), limit (3.8), it follows that $\frac{1}{\delta} E\left[\left\|Y_{s+\delta}-Y_{s}\right\|^{2}\right] \quad \underset{\delta \rightarrow 0}{\longrightarrow} \quad \theta, s \in[0, \infty)$. Relations (3.18) and (3.19) together with Lemma 3.4 show that $\frac{1}{\delta} \int_{0}^{t}\left\|Y_{s+\delta}-Y_{s}\right\|^{2} d s \quad \overrightarrow{\delta \rightarrow 0} \quad \theta t$ in probability. Thanks to the fact that $[0, \infty) \ni t \rightarrow \frac{1}{\delta} \int_{0}^{t}\left\|Y_{s+\delta}-Y_{s}\right\|^{2} d s$ is increasing for all $\delta$, Lemma 3.1 in 21] implies even

$$
\frac{1}{\delta} \int_{0}^{t}\left\|Y_{s+\delta}-Y_{s}\right\|^{2} d s \quad \underset{\delta \rightarrow 0}{\longrightarrow} \quad \theta t \quad \text { ucp on }[0, \infty)
$$

Recalling $A_{t}=\sum_{i=1}^{\infty} e^{-\lambda_{i} t} G_{i}(0) \cdot S_{i}$ and defining $(Z+A)_{t}:=Z_{t}+A_{t}, t \geq 0$, as in (3.13) we may conclude

$$
\begin{aligned}
& \left|\frac{1}{\delta} \int_{0}^{t}\left\|X_{s+\delta}-X_{s}\right\|^{2} d s-\frac{1}{\delta} \int_{0}^{t}\left\|Y_{s+\delta}-Y_{s}\right\|^{2} d s\right| \\
& \quad \leq \frac{2}{\delta} \int_{0}^{t}\left\|Y_{s+\delta}-Y_{s}\right\|\left\|(Z+A)_{s+\delta}-(Z+A)_{s}\right\| d s+\frac{1}{\delta} \int_{0}^{t}\left\|(Z+A)_{s+\delta}-(Z+A)_{s}\right\|^{2} d s \\
& =\frac{2}{\delta} \int_{0}^{\delta} \sum_{k=1}^{\left[\frac{t-a}{\delta}\right]+1}\left\|Y_{a+k \delta}-Y_{a+(k-1) \delta}\right\|\left\|(Z+A)_{a+k \delta}-(Z+A)_{a+(k-1) \delta}\right\| d a \\
& \quad+\frac{1}{\delta} \int_{0}^{\delta} \sum_{k=1}^{\left[\frac{t-a}{\delta}\right]+1}\left\|(Z+A)_{a+k \delta}-(Z+A)_{a+(k-1) \delta}\right\|^{2} d a .
\end{aligned}
$$

The two items on the right hand side tend to zero ucp on $t \in[0, \infty)$ by following the method of (3.18)-(3.20) with Markov's instead of Chebychev's inequality and applying the estimates of the proof of Proposition 3.3. The claim follows. 


\section{Tensor quadratic variation}

Let us use the notation of the previous section. In addition, let $\|\cdot\|_{\pi}$ denote the projective norm with respect to the tensor product $L^{1}\left([0,1] ; \mathbb{R}^{d}\right) \otimes L^{1}\left([0,1] ; \mathbb{R}^{d}\right)$, i.e. the norm in the Banach space $L^{1}\left([0,1] ; \mathbb{R}^{d}\right) \widehat{\otimes}_{\pi} L^{1}\left([0,1] ; \mathbb{R}^{d}\right)$. We recall that the algebraic tensor product $L^{1}\left([0,1] ; \mathbb{R}^{d}\right) \otimes L^{1}\left([0,1] ; \mathbb{R}^{d}\right)$ denotes all elements of the form $\sum_{i=1}^{n} x_{i} \otimes y_{i}$ where $x_{i}, y_{i} \in L^{1}\left([0,1] ; \mathbb{R}^{d}\right), n \in \mathbb{N}$. For an element $u \in L^{1}\left([0,1] ; \mathbb{R}^{d}\right) \otimes L^{1}\left([0,1] ; \mathbb{R}^{d}\right)$ the projective norm is defined by

$$
\|u\|_{\pi}:=\inf \left\{\sum_{i=1}^{n}\left\|x_{i}\right\|_{L^{1}}\left\|y_{i}\right\|_{L^{1}}: u=\sum_{i=1}^{n} x_{i} \otimes y_{i}, \quad x_{i}, y_{i} \in L^{1}\left([0,1] ; \mathbb{R}^{d}\right)\right\} .
$$

The space $L^{1}\left([0,1] ; \mathbb{R}^{d}\right) \widehat{\otimes}_{\pi} L^{1}\left([0,1] ; \mathbb{R}^{d}\right)$ is the completion of $L^{1}\left([0,1] ; \mathbb{R}^{d}\right) \otimes L^{1}\left([0,1] ; \mathbb{R}^{d}\right)$ with respect to the projective norm. Recall that there is an isometric isomorphism $i: L^{1}\left([0,1] ; \mathbb{R}^{d}\right) \widehat{\otimes}_{\pi} L^{1}\left([0,1] ; \mathbb{R}^{d}\right) \rightarrow$ $L^{1}\left([0,1] \times[0,1] ; \mathbb{R}^{d^{2}}\right)$ given by

$$
(i \circ f \otimes g)(u, v)=\left(i \circ f \widehat{\otimes}_{\pi} g\right)(u, v):=f(u) \otimes g(v), \quad f, g \in L^{1}\left([0,1] ; \mathbb{R}^{d}\right), \quad(u, v) \in[0,1]^{2} .
$$

The reference measure $\mu$ on $\left([0,1]^{2}, \mathcal{B}\left([0,1]^{2}\right)\right)$ is the Lebesgue measure. This also says that $f(u) \otimes g(v)$ is identified with $\left((f(u))_{i}(g(v))_{j}\right)_{i, j=1 \ldots d}$. Here $\mathbb{R}^{d^{2}}$ is endowed with the norm $\|\mathbf{x}\|_{1,1}:=\sum_{i, j=1}^{d}\left|x_{i j}\right|$ where $\mathbf{x}=\left(x_{11}, \ldots, x_{d d}\right), x_{11}, \ldots, x_{d d} \in \mathbb{R}$. Similarly for the norm in $\mathbb{R}^{d}$ we denote $\|\mathbf{y}\|_{1}:=\sum_{i=1}^{d}\left|y_{i}\right|$, where $\mathbf{y}=\left(y_{1}, \ldots, y_{d}\right), y_{1}, \ldots, y_{d} \in \mathbb{R}$. We also introduce the notation $x^{\otimes^{2}} \equiv x \otimes x$ which will be used below.

In this section we aim to determine the tensor valued quadratic variation

$$
[X]_{t}^{\otimes}:=\lim _{\delta \rightarrow 0} \int_{0}^{t} \frac{\left(X_{u+\delta}-X_{u}\right) \otimes\left(X_{u+\delta}-X_{u}\right)}{\delta} d u
$$

in the ucp sense with respect to the $\pi$-norm. Below we take advantage of [7], and [8], however we would also like to refer to the classical work of [17]. Let $\xi_{i}, i \in \mathbb{N}$, be independent standard normal random variables and define

$$
\Theta:=2 E\left[\left(\sum_{i=1}^{\infty} \lambda_{i}^{\frac{1}{2}} \xi_{i} S_{i}\right) \otimes\left(\sum_{i^{\prime}=1}^{\infty} \lambda_{i^{\prime}}^{\frac{1}{2}} \xi_{i^{\prime}} S_{i^{\prime}}\right)\right]
$$

provided that this expression exists in $L^{1}\left([0,1] ; \mathbb{R}^{d}\right) \widehat{\otimes}_{\pi} L^{1}\left([0,1] ; \mathbb{R}^{d}\right)$. To ensure compatibility with [7, [8], and [12] all integrals with Banach space valued integrands, as for example in the definitions of $[X]^{\otimes}$ and $\Theta$, are Bochner integrals.

Proposition 4.1. Suppose (3.9) and (3.10), i.e.

$$
\sum_{m=0}^{\infty} 2^{-\frac{m}{2}} \cdot \lambda_{d 2^{m+1}} \cdot\left(\max _{d 2^{m}+1<i \leq d 2^{m+1}}\left|G_{i}(0)\right|+m^{\frac{1}{2}}\right)<\infty .
$$

Then $\Theta$ is well-defined by (4.1) and we have in the norm of $L^{1}\left([0,1] ; \mathbb{R}^{d}\right) \widehat{\otimes}_{\pi} L^{1}\left([0,1] ; \mathbb{R}^{d}\right)$

$$
\frac{1}{\delta} \int_{0}^{t}\left(X_{s+\delta}-X_{s}\right) \otimes\left(X_{s+\delta}-X_{s}\right) d s \quad \underset{\delta \rightarrow 0}{\longrightarrow} \quad t \Theta \quad \text { ucp on } t \in[0, \infty) .
$$

Proof. Using the independence of the $\xi_{i}, i \in \mathbb{N}$, we obtain

$$
\begin{aligned}
& E\left[\left\|i \circ\left(\sum_{i=1}^{\infty} \lambda_{i}^{\frac{1}{2}} \xi_{i} S_{i}\right) \otimes\left(\sum_{i^{\prime}=1}^{\infty} \lambda_{i^{\prime}}^{\frac{1}{2}} \xi_{i^{\prime}} S_{i^{\prime}}\right)\right\|_{L^{1}\left([0,1]^{2} ; \mathbb{R}^{d^{2}}\right)}\right] \leq\left(\sum_{i=1}^{\infty} \lambda_{i}^{\frac{1}{2}}\left(E\left[\xi_{i}^{2}\right]\right)^{\frac{1}{2}}\left\|S_{i}\right\|_{L^{1}\left([0,1] ; \mathbb{R}^{d}\right)}\right)^{2} \\
& \leq\left(\sum_{i=1}^{d} \lambda_{i}^{\frac{1}{2}}+\sum_{m=0}^{\infty} 2^{-\frac{m+4}{2}} \lambda_{d 2^{m+1}}^{\frac{1}{2}}\right)^{2}<\infty
\end{aligned}
$$


where the second line follows from (3.10). Thus the existence and representation of $\Theta$ defined in (4.1) follows from [12], Theorem 10.2.

Without further reference, we will use the inequality $P\left(\sum_{i}\left|\zeta_{i}\right| \geq a\right) \leq \sum_{i} P\left(\left|\zeta_{i}\right| \geq b_{i} a\right)$ several times in the proof. Here the $\zeta_{i}$ are arbitrary random variables, $b_{i}>0$ with $\sum_{i} b_{i}=1$, and $a>0$.

Step 1: Let us introduce some of the important objects in the proof. First we recall that $X_{t}=$ $Y_{t}+Z_{t}+A_{t}, t \geq 0$, where the individual items $Y, Z$, and $A$ are defined in the beginning of Section 3 , Let

$$
\eta_{t}(\delta):=\frac{1}{\delta} \int_{0}^{\delta}\left(Y_{s}-Y_{0}\right)^{\otimes^{2}} d s+\frac{1}{\delta} \int_{0}^{t}\left(Y_{(s+\delta) \wedge t}-Y_{s}\right)^{\otimes^{2}} d s-t \Theta, \quad t \geq \delta \geq 0 .
$$

Among other things, this says

$$
\begin{aligned}
& \frac{1}{\delta} \int_{0}^{t}\left(Y_{s+\delta}-Y_{s}\right)^{\otimes^{2}} d s-t \Theta=\eta_{t}(\delta)+\frac{1}{\delta}\left(-\int_{0}^{\delta}\left(Y_{s}-Y_{0}\right)^{\otimes^{2}} d s+\int_{t-\delta}^{t}\left(Y_{s+\delta}-Y_{t}\right)^{\otimes^{2}} d s\right. \\
& \left.\quad+\int_{t-\delta}^{t}\left(Y_{s+\delta}-Y_{t}\right) \otimes\left(Y_{t}-Y_{s}\right) d s+\int_{t-\delta}^{t}\left(Y_{t}-Y_{s}\right) \otimes\left(Y_{s+\delta}-Y_{t}\right) d s\right) \\
& =: \eta_{t}(\delta)+\zeta_{t}(\delta) \equiv \eta_{t}+\zeta_{t} .
\end{aligned}
$$

We look at the second item of the right-hand side. Taking into consideration the above isometry and Fubini's theorem we get for $T \geq \delta$ and $\varepsilon>0$

$$
P\left(\sup _{0 \leq t \leq T}\left\|\delta \zeta_{t}\right\|_{\pi} \geq \frac{1}{4} \delta \varepsilon\right) \leq P\left(\sup _{0 \leq t \leq T, 0 \leq \gamma \leq \delta}\left\|Y_{t+\gamma}-Y_{t}\right\|_{L^{1}\left([0,1] ; \mathbb{R}^{d}\right)}^{2} \geq \frac{1}{16} \varepsilon\right)=: g(\delta ; \varepsilon) .
$$

Step 2: We examine $g(\delta ; \varepsilon)$. According to Lévy's characterization of Brownian motion we have

$$
Y_{t+\gamma}-Y_{t}=\sum_{i=1}^{\infty}\left(\left(2 \lambda_{i}\right)^{\frac{1}{2}} V_{i}(t+\gamma) S_{i}-\left(2 \lambda_{i}\right)^{\frac{1}{2}} V_{i}(t) S_{i}\right)
$$

where $V_{i}, i \in \mathbb{N}$, are independent one-dimensional Wiener processes. We obtain

$$
g(\delta ; \varepsilon) \leq \sum_{i=1}^{\infty} P\left(\sup _{0 \leq t \leq T, 0 \leq \gamma \leq \delta}\left\|S_{i}\right\|_{L^{1}}\left(2 \lambda_{i}\right)^{\frac{1}{2}}\left|V_{i}(t+\gamma)-V_{i}(t)\right| \geq \frac{b_{i}}{4} \varepsilon^{\frac{1}{2}}\right)
$$

where we choose

$$
b_{i}:=\frac{4(\ln (i+1))^{\frac{1}{2}} \cdot\left(2 \lambda_{i}\right)^{\frac{1}{2}}\left\|S_{i}\right\|_{L^{1}}}{4 \sum_{i^{\prime}=1}^{\infty}\left(\ln \left(i^{\prime}+1\right)\right)^{\frac{1}{2}} \cdot\left(2 \lambda_{i^{\prime}}\right)^{\frac{1}{2}}\left\|S_{i^{\prime}}\right\|_{L^{1}}} \equiv \frac{4(\ln (i+1))^{\frac{1}{2}} \cdot\left(2 \lambda_{i}\right)^{\frac{1}{2}}\left\|S_{i}\right\|_{L^{1}}}{D}, \quad i \in \mathbb{N} .
$$

We note that the denominator $D$ is finite by $\left\|S_{i}\right\|_{L^{1}} \equiv\left\|S_{i}\right\|_{L^{1}\left([0,1] ; \mathbb{R}^{d}\right)}=2^{-\frac{3 m-4}{2}}$ if $d 2^{m}+1 \leq i \leq d 2^{m+1}$ for $m \in\{0,1,2, \ldots\}$ and hypothesis (3.10). Next we use the subsequent inequality which follows from Lemma 2.1 of [3] by choosing $\mu=1$ and scaling. There exists $C>0$ independent of $v$ and $\delta$ such that

$$
P\left(\sup _{0 \leq t \leq T, 0 \leq \gamma \leq \delta}\left|V_{i}(t+\gamma)-V_{i}(t)\right| \geq v \delta^{\frac{1}{2}}\right) \leq \frac{C}{\delta} \exp \left\{-\frac{v^{2}}{3}\right\}, \quad v>0 .
$$

We obtain

$$
P\left(\sup _{0 \leq t \leq T, 0 \leq \gamma \leq \delta}\left(2 \lambda_{i}\right)^{\frac{1}{2}}\left\|S_{i}\right\|_{L^{1}} \cdot\left|V_{i}(t+\gamma)-V_{i}(t)\right| \geq \frac{b_{i}}{4} \varepsilon^{\frac{1}{2}}\right) \leq \frac{C}{\delta} \exp \left\{-\frac{\ln (i+1) \cdot \varepsilon}{3 D^{2} \delta}\right\}
$$

for some $C>0$ independent of $\varepsilon, i$, and $\delta$. Applying (4.5) and (4.6) to (4.4) yields

$$
P\left(\sup _{0 \leq t \leq T, 0 \leq \gamma \leq \delta}\left\|Y_{t+\gamma}-Y_{t}\right\|_{L^{1}\left([0,1] ; \mathbb{R}^{d}\right)} \geq \frac{1}{4} \varepsilon^{\frac{1}{2}}\right) \leq \frac{C}{\delta} \sum_{i=1}^{\infty} \exp \left\{-\frac{\ln (i+1) \cdot \varepsilon}{3 D^{2} \delta}\right\} .
$$


It follows that

$$
g(\delta ; \varepsilon) \quad \underset{\delta \rightarrow 0}{\longrightarrow} 0
$$

for all $\varepsilon>0$. Using similar arguments we get

$$
\begin{aligned}
& P\left(\sup _{0 \leq t \leq \delta}\left\|\int_{0}^{t}\left(Y_{s+\delta}-Y_{s}\right)^{\otimes^{2}} d s-\delta t \Theta\right\|_{\pi} \geq \frac{1}{2} \delta \varepsilon\right) \\
& \quad \leq P\left(\sup _{0 \leq s, t \leq \delta}\left\|Y_{t}-Y_{s}\right\|_{L^{1}\left([0,1] ; \mathbb{R}^{d}\right)}^{2}+\delta\|\Theta\|_{\pi} \geq \frac{1}{2} \varepsilon\right)=: h(\delta ; \varepsilon) \quad \overrightarrow{\delta \rightarrow 0} \quad 0 .
\end{aligned}
$$

Step 3: Taking our attention back to $\eta_{t}$, for $t>u \geq \delta$ we note that

$$
\begin{aligned}
\eta_{t}= & \frac{1}{\delta} \int_{0}^{\delta}\left(Y_{s}-Y_{0}\right)^{\otimes^{2}} d s+\frac{1}{\delta} \int_{0}^{u-\delta}\left(Y_{s+\delta}-Y_{s}\right)^{\otimes^{2}} d s+\frac{1}{\delta} \int_{u-\delta}^{u}\left(Y_{u}-Y_{s}\right)^{\otimes^{2}} d s \\
& +\frac{1}{\delta} \int_{u-\delta}^{u}\left(Y_{(s+\delta) \wedge t}-Y_{u}\right)^{\otimes^{2}} d s+\frac{1}{\delta} \int_{u-\delta}^{u}\left(Y_{(s+\delta) \wedge t}-Y_{u}\right) \otimes\left(Y_{u}-Y_{s}\right) d s \\
& +\frac{1}{\delta} \int_{u-\delta}^{u}\left(Y_{u}-Y_{s}\right) \otimes\left(Y_{(s+\delta) \wedge t}-Y_{u}\right) d s+\frac{1}{\delta} \int_{u}^{t}\left(Y_{(s+\delta) \wedge t}-Y_{s}\right)^{\otimes^{2}} d s-t \Theta \\
= & \eta_{u}+\frac{1}{\delta} \int_{u-\delta}^{u}\left(Y_{(s+\delta) \wedge t}-Y_{u}\right)^{\otimes^{2}} d s+\frac{1}{\delta} \int_{u-\delta}^{u}\left(Y_{(s+\delta) \wedge t}-Y_{u}\right) \otimes\left(Y_{u}-Y_{s}\right) d s \\
& +\frac{1}{\delta} \int_{u-\delta}^{u}\left(Y_{u}-Y_{s}\right) \otimes\left(Y_{(s+\delta) \wedge t}-Y_{u}\right) d s+\frac{1}{\delta} \int_{u}^{t}\left(Y_{(s+\delta) \wedge t}-Y_{s}\right)^{\otimes^{2}} d s-(t-u) \Theta .
\end{aligned}
$$

By the definition of $\Theta$ and the above isomorphism it follows that $E\left[\eta_{t} \mid \mathcal{F}_{u}\right]=E\left[\eta_{t}-\eta_{u} \mid \mathcal{F}_{u}\right]+\eta_{u}=\eta_{u}$, $t>u \geq \delta$. In other words, $\eta_{t}, t \geq \delta$, is a martingale. For well-definiteness see again [12], Theorem 10.2.

Using (4.2), (4.3), (4.9), and Doob's inequality we obtain for $T \geq \delta$ and $\varepsilon>0$

$$
\begin{aligned}
& P\left(\sup _{0 \leq t \leq T}\left\|\int_{0}^{t}\left(Y_{s+\delta}-Y_{s}\right)^{\otimes^{2}} d s-\delta t \Theta\right\|_{\pi} \geq \delta \varepsilon\right) \\
& \leq P\left(\sup _{0 \leq t \leq \delta}\left\|\int_{0}^{t}\left(Y_{s+\delta}-Y_{s}\right)^{\otimes^{2}} d s-\delta t \Theta\right\|_{\pi} \geq \frac{1}{2} \delta \varepsilon\right) \\
& \quad+P\left(\sup _{\delta \leq t \leq T}\left\|\int_{0}^{t}\left(Y_{s+\delta}-Y_{s}\right)^{\otimes^{2}} d s-\delta t \Theta\right\|_{\pi} \geq \frac{1}{2} \delta \varepsilon\right) \\
& \leq h(\delta ; \varepsilon)+P\left(\sup _{\delta \leq t \leq T}\left\|\delta \eta_{t}\right\|_{\pi} \geq \frac{1}{4} \delta \varepsilon\right)+g(\delta ; \varepsilon) \\
& \quad \leq h(\delta ; \varepsilon)+\frac{16}{\varepsilon^{2} \delta^{2}} E\left[\left\|\delta \eta_{T}\right\|_{\pi}^{2}\right]+g(\delta ; \varepsilon) \\
& \quad\left[\left\|\left(\int_{0}^{T}\left(Y_{s+\delta}-Y_{s}\right)^{\otimes^{2}} d s-\delta T \Theta\right)-\delta \zeta_{T}\right\|_{\pi}^{2}\right]+g(\delta ; \varepsilon)+h(\delta ; \varepsilon) .
\end{aligned}
$$

Next we introduce

$$
\langle Y\rangle_{a}^{\otimes} \equiv\langle Y\rangle_{a, T}^{\otimes}(\delta):=\sum_{k=1}^{\left[\frac{T-a}{\delta}\right]+1}\left[\left(Y_{a+k \delta}-Y_{a+(k-1) \delta}\right)^{\otimes^{2}}-\delta \Theta\right]
$$

and note that $\langle Y\rangle_{a}^{\otimes}$ is almost surely an element of $\in L^{1}\left([0,1] ; \mathbb{R}^{d}\right) \widehat{\otimes}_{\pi} L^{1}\left([0,1] ; \mathbb{R}^{d}\right)$. From (4.10) we 
obtain

$$
\begin{aligned}
P & \left(\sup _{0 \leq t \leq T}\left\|\int_{0}^{t}\left(Y_{s+\delta}-Y_{s}\right)^{\otimes^{2}} d s-\delta t \Theta\right\|_{\pi} \geq \delta \varepsilon\right) \\
& =\frac{32}{\varepsilon^{2} \delta^{2}} E\left[\left\|\int_{0}^{\delta}\langle Y\rangle_{a}^{\otimes} d a\right\|_{\pi}^{2}\right]+\frac{32}{\varepsilon^{2} \delta^{2}} E\left[\left\|\delta \zeta_{T}\right\|_{\pi}^{2}\right]+g(\delta ; \varepsilon)+h(\delta ; \varepsilon) \\
& \leq \frac{32}{\delta \varepsilon^{2}} \int_{0}^{\delta} E\left[\left\|\langle Y\rangle_{a}^{\otimes}\right\|_{\pi}^{2}\right] d a+\frac{32}{\varepsilon^{2} \delta^{2}} E\left[\left\|\delta \zeta_{T}\right\|_{\pi}^{2}\right]+g(\delta ; \varepsilon)+h(\delta ; \varepsilon) .
\end{aligned}
$$

Step 4: In this step we examine the expression $E\left[\left\|\langle Y\rangle_{a}^{\otimes}\right\|_{\pi}^{2}\right]$. We do this by first applying the isometry $i: L^{1}\left([0,1] ; \mathbb{R}^{d}\right) \widehat{\otimes}_{\pi} L^{1}\left([0,1] ; \mathbb{R}^{d}\right) \rightarrow L^{1}\left([0,1] \times[0,1] ; \mathbb{R}^{d^{2}}\right)$, followed by the Schwarz inequality, and Fubini's theorem to obtain

$$
\begin{aligned}
E & {\left[\left\|\langle Y\rangle_{a}^{\otimes}\right\|_{\pi}^{2}\right]=E\left[\int_{\left([0,1]^{2}\right)^{2}}\left\|\left(i \circ\langle Y\rangle_{a}^{\otimes}\right)(u, v)\right\|_{1,1} \cdot\left\|\left(i \circ\langle Y\rangle_{a^{\prime}}^{\otimes}\right)\left(u^{\prime}, v^{\prime}\right)\right\|_{1,1} d \mu(u, v) d \mu\left(u^{\prime}, v^{\prime}\right)\right] } \\
& =\int_{\left([0,1]^{2}\right)^{2}} E\left[\left\|\left(i \circ\langle Y\rangle_{a}^{\otimes}\right)(u, v)\right\|_{1,1} \cdot\left\|\left(i \circ\langle Y\rangle_{a^{\prime}}^{\otimes}\right)\left(u^{\prime}, v^{\prime}\right)\right\|_{1,1}\right] d \mu(u, v) d \mu\left(u^{\prime}, v^{\prime}\right) \\
& \leq\left(\int_{[0,1]^{2}}\left(E\left[\left\|\left(i \circ\langle Y\rangle_{a}^{\otimes}\right)(u, v)\right\|_{1,1}^{2}\right]\right)^{\frac{1}{2}} d \mu(u, v)\right)^{2} .
\end{aligned}
$$

Taking into consideration the independence of the increments of $Y$ we get

$$
\begin{aligned}
E & {\left[\left\|\left(i \circ\langle Y\rangle_{a}^{\otimes}\right)(u, v)\right\|_{1,1}^{2}\right]=\sum_{k=1}^{\left[\frac{t-a}{\delta}\right]+1} E\left[\left\|\left(i \circ\left(\left(Y_{a+k \delta}-Y_{a+(k-1) \delta}\right)^{\otimes^{2}}-\delta \Theta\right)\right)(u, v)\right\|_{1,1}^{2}\right] } \\
= & \sum_{k=1}^{\left[\frac{t-a}{\delta}\right]+1} E\left[\|\left(Y_{a+k \delta}-Y_{a+(k-1) \delta}\right)(u) \otimes\left(Y_{a+k \delta}-Y_{a+(k-1) \delta}\right)(v)\right. \\
& \left.-E\left[\left(Y_{a+k \delta}-Y_{a+(k-1) \delta}\right)(u) \otimes\left(Y_{a+k \delta}-Y_{a+(k-1) \delta}\right)(v)\right] \|_{1,1}^{2}\right] \\
\leq & \sum_{k=1}^{\left[\frac{t-a}{\delta}\right]+1} E\left[\left\|\left(Y_{a+k \delta}-Y_{a+(k-1) \delta}\right)(u) \otimes\left(Y_{a+k \delta}-Y_{a+(k-1) \delta}\right)(v)\right\|_{1,1}^{2}\right] .
\end{aligned}
$$

It follows from the Schwarz inequality that

$$
\begin{aligned}
E & {\left[\left\|\left(i \circ\langle Y\rangle_{a}^{\otimes}\right)(u, v)\right\|_{1,1}^{2}\right] } \\
& \leq \sum_{k=1}^{\left[\frac{t-a}{\delta}\right]+1}\left(E\left[\left\|\left(Y_{a+k \delta}-Y_{a+(k-1) \delta}\right)(u)\right\|_{1}^{4}\right]\right)^{\frac{1}{2}} \cdot\left(E\left[\left\|\left(Y_{a+k \delta}-Y_{a+(k-1) \delta}\right)(v)\right\|_{1}^{4}\right]\right)^{\frac{1}{2}} .
\end{aligned}
$$

We recall from the proof of Lemma 3.1 that $Y_{a+k \delta}-Y_{a+(k-1) \delta}=\sum_{i=1}^{\infty} \Delta_{i, a+k \delta} Y \cdot S_{i}$ where $\Delta_{i, a+k \delta}$ is $N\left(0,2 \lambda_{i} \delta\right)$-distributed, $i \in \mathbb{N}$, and thus by relation (3.6) and hypothesis (3.10)

$$
E\left[\left\|\left(Y_{a+k \delta}-Y_{a+(k-1) \delta}\right)(u)\right\|_{1}^{4}\right] \leq C \delta^{2}
$$

for some $C>0$ independent of $u \in[0,1]$. By (4.2) and similar calculations it follows that

$$
E\left[\left\|\zeta_{T}\right\|_{\pi}^{2}\right] \underset{\delta \rightarrow 0}{\longrightarrow} 0 .
$$


Now (4.11) together with (4.8), (4.9), (4.15), and (4.12)-(4.14) imply

$$
\int_{0}^{t}\left(Y_{s+\delta}-Y_{s}\right)^{\otimes^{2}} d s \quad \underset{\delta \rightarrow 0}{\longrightarrow} t \Theta \quad \text { ucp on }[0, \infty)
$$

in the norm of $L^{1}\left([0,1] ; \mathbb{R}^{d}\right) \widehat{\otimes}_{\pi} L^{1}\left([0,1] ; \mathbb{R}^{d}\right)$.

Step 5: It remains to show that

$$
\left\|\frac{1}{\delta} \int_{0}^{t}\left(X_{a+\delta}-X_{a}\right)^{\otimes^{2}} d a-\frac{1}{\delta} \int_{0}^{t}\left(Y_{a+\delta}-Y_{a}\right)^{\otimes^{2}} d a\right\|_{\pi} \quad \underset{\delta \rightarrow 0}{\longrightarrow} \quad 0 \quad \text { ucp on }[0, \infty) .
$$

Using the relation $X_{t}=Y_{t}+Z_{t}+A_{t}$, as defined in Section 3 we obtain

$$
\begin{aligned}
\| & \frac{1}{\delta} \int_{0}^{t}\left(X_{s+\delta}-X_{s}\right)^{\otimes^{2}}-\left(Y_{s+\delta}-Y_{s}\right)^{\otimes^{2}} d s \|_{\pi} \\
=\| & \frac{1}{\delta} \int_{0}^{t}\left(Y_{s+\delta}+Z_{s+\delta}+A_{s+\delta}-Y_{s}-Z_{s}-A_{s}\right)^{\otimes^{2}}-\left(Y_{s+\delta}-Y_{s}\right)^{\otimes^{2}} d s \|_{\pi} \\
=\| & \frac{1}{\delta} \int_{0}^{t}\left(Y_{s+\delta}-Y_{s}\right) \otimes\left(Z_{s+\delta}+A_{s+\delta}-Z_{s}-A_{s}\right)+\left(Z_{s+\delta}+A_{s+\delta}-Z_{s}-A_{s}\right) \otimes\left(Y_{s+\delta}-Y_{s}\right) \\
& \quad+\left(Z_{s+\delta}+A_{s+\delta}-Z_{s}-A_{s}\right)^{\otimes^{2}} d s \|_{\pi} .
\end{aligned}
$$

Using the isometry of $i: L^{1}\left([0,1] ; \mathbb{R}^{d}\right) \widehat{\otimes}_{\pi} L^{1}\left([0,1] ; \mathbb{R}^{d}\right) \rightarrow L^{1}\left([0,1]^{2} ; \mathbb{R}^{d^{2}}\right)$ and the triangle inequality we get the estimate

$$
\begin{aligned}
& \left\|\frac{1}{\delta} \int_{0}^{t}\left(X_{s+\delta}-X_{s}\right)^{\otimes^{2}}-\left(Y_{s+\delta}-Y_{s}\right)^{\otimes^{2}} d s\right\|_{\pi} \\
& \quad \leq \frac{1}{\delta} \int_{0}^{t} 2\left\|Y_{s+\delta}-Y_{s}\right\|_{L^{1}}\left\|(Z+A)_{s+\delta}-(Z+A)_{s}\right\|_{L^{1}}+\left\|(Z+A)_{s+\delta}-(Z+A)_{s}\right\|_{L^{1}}^{2} d s
\end{aligned}
$$

with $\|\cdot\|_{L^{1}}$ abbreviating the norm in $L^{1}\left([0,1] ; \mathbb{R}^{d}\right)$. This is precisely the expression (3.21) of the proof of Proposition 3.5 (b). The proof of Proposition 3.5 (b) shows that under the assumptions (3.9) and (3.10) this expression tends to $0 \mathrm{ucp}$ as $\delta \rightarrow 0$. The claim follows.

\section{Itô's formula}

According to Lemma 3.1 (b) and relation (3.12), the processes $Y$ and $Z$ are quadratically integrable provided that (3.9) and (3.10) hold, choose $\tau=\left\{0=t_{0}, t_{1}=T\right\}$ for this. Thus, depending on (quadratic) integrability of $\sum_{i=1}^{\infty} G_{i}(0) S_{i}$, the process $X$ is a (quadratically) integrable semimartingale with decomposition $X=Y+(Z+A), Y$ being the martingale part. In order to establish an Itô formula for the process $X$, one could think of applying the Itô formula in Banach spaces given by [11, if possible. However, having established the tensor quadratic variation in Section 4, it is more natural and more direct to take advantage of the Itô formula in Banach spaces corresponding to the stochastic calculus of regularization, see [7] and [8].

To ease the notation in this section we denote $B:=L^{1}\left([0,1] ; \mathbb{R}^{d}\right)$. Below we use the pairing dualities $B^{*}\langle\cdot, \cdot\rangle_{B}$ and $\left.{ }_{\left(B \widehat{\otimes}_{\pi} B\right)^{*}}\langle\cdot, \cdot\rangle_{(B} \widehat{\otimes}_{\pi} B\right)^{* *}$ in the sense and notation of [7] and [8]. Using the standard identification of the dual spaces, we recall that for $f \in L^{1}\left([0,1] ; \mathbb{R}^{d}\right)=B, g^{*} \in B^{*}$, and some representing element $g \in L^{\infty}\left([0,1] ; \mathbb{R}^{d}\right) \cong B^{*}$ of $g^{*}$ we have

$$
B^{*}\left\langle g^{*}, f\right\rangle_{B}=\int_{0}^{1} f(u) \cdot g(u) d u .
$$




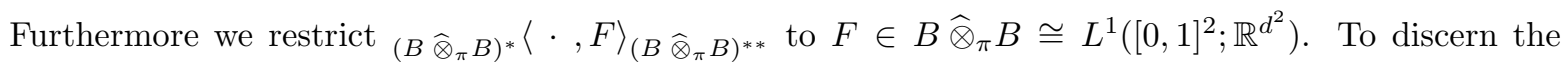
different scalar products we will use the symbol - to denote the scalar product in $\mathbb{R}^{d^{2}}$. For $G^{*} \in$ $\left(B \widehat{\otimes}_{\pi} B\right)^{*}$ and some representing element $G \in L^{\infty}\left([0,1]^{2} ; \mathbb{R}^{d^{2}}\right) \cong\left(B \widehat{\otimes}_{\pi} B\right)^{*}$ the pairing duality becomes

$$
\left(B \widehat{\otimes}_{\pi} B\right)^{*}\left\langle G^{*}, F\right\rangle_{\left(B \widehat{\otimes}_{\pi} B\right)^{* *}}=\int F(u, v) \cdot G(u, v) d u d v .
$$

Definition 5.1. Let $\left(X_{t}\right)_{t \in[0, T]}$ and $\left(Y_{t}\right)_{t \in[0, T]}$ be continuous $B$-valued, respectively $B^{*}$-valued stochastic processes. The forward integral of $Y$ with respect to $X$ denoted by $\int_{0}^{t} B^{*}\left\langle Y_{s}, d X_{s}\right\rangle_{B}$ is defined as the limit

$$
\int_{0}^{t} B^{*}\left\langle Y_{s}, d X_{s}\right\rangle_{B}:=\lim _{\varepsilon \rightarrow 0} \int_{0}^{t}\left\langle Y_{s}, \frac{X_{s+\varepsilon}-X_{s}}{\varepsilon}\right\rangle_{B} d s
$$

in probability if it exists.

Definition 5.2. Let $F$ be a mapping $F:[0, T] \times B \rightarrow \mathbb{R}$. We say that $F$ is of Fréchet class $C^{1,2}$ (in symbols $F \in C^{1,2}$ ) if $F$ is one time continuously Fréchet differentiable and two times continuously Fréchet differentiable in the second argument. That is, denoting the Fréchet derivative with respect to the second variable by $D$, for every $t \in[0, T]$ we have $D F(t, \cdot): B \rightarrow B^{*}$ and $D^{2} F(t, \cdot): B \rightarrow\left(B \widehat{\otimes}_{\pi} B\right)^{*}$ continuously.

Theorem 5.3. Suppose (3.9) and (3.10), i.e.

$$
\sum_{m=0}^{\infty} 2^{-\frac{m}{2}} \cdot \lambda_{d 2^{m+1}} \cdot\left(\max _{d 2^{m}+1<i \leq d 2^{m+1}}\left|G_{i}(0)\right|+m^{\frac{1}{2}}\right)<\infty .
$$

Let $\left(X_{t}\right)_{t \in[0, T]}$ be given by (2.3), i.e.

$$
X_{t}=\sum_{i=1}^{\infty} G_{i}\left(\lambda_{i} t\right) \cdot S_{i}, \quad t \geq 0 .
$$

Furthermore denote $B=L^{1}\left([0,1] ; \mathbb{R}^{d}\right)$, and let $F \in C^{1,2}$. Then

(a) For every $t \in[0, T]$ the forward integral $\int_{0}^{t} B^{*}\left\langle D F\left(s, X_{s}\right), d X_{s}\right\rangle_{B}$ exists.

(b) We have the Itô formula

$$
\begin{aligned}
& F\left(t, X_{t}\right)=F\left(0, X_{0}\right)+\int_{0}^{t} \frac{\partial}{\partial s} F\left(s, X_{s}\right) d s+\int_{0}^{t} B^{*}\left\langle D F\left(s, X_{s}\right), d X_{s}\right\rangle_{B} \\
& +\frac{1}{2} \int_{0}^{t}\left(B \widehat{\otimes}_{\pi} B\right)^{*}\left\langle D^{2} F\left(s, X_{s}\right), \Theta\right\rangle_{\left(B \widehat{\otimes}_{\pi} B\right)^{* *}} d s,
\end{aligned}
$$

where $\Theta$ is given by (4.1).

Proof. (a) Due to the existence of a scalar and tensor quadratic variation for $X_{t}$ we may apply Proposition 3.15 of [8] and Theorem 6.3 of [7] from which the statement follows.

(b) This is an immediate consequence of Proposition 4.1 above, and Theorem 6.3 of [7] together with Remark 6.2 of [7].

Now let us specify the Itô formula to cylindrical functions $F$ of type $F(s, \gamma)=f\left(s ;\left\langle S_{1}, \gamma\right\rangle, \ldots,\left\langle S_{k}, \gamma\right\rangle\right)$, $f \in C_{0}^{\infty}\left(\mathbb{R}^{k+1}\right), s \geq 0, \gamma \in C_{0}\left([0,1] ; \mathbb{R}^{d}\right)$. Here, $S_{i}$ and $\left\langle S_{i}, \gamma\right\rangle$ are given by $S_{i}(s):=\int_{0}^{s} g_{i}(u) d u$ and $\left\langle S_{i}, \gamma\right\rangle:=\int_{0}^{1} g_{i}(u) d \gamma_{u}$, cf. (1.7). Since such functions $F$ are discontinuous if $\gamma$ is considered as an element belonging to $L^{1}\left([0,1] ; \mathbb{R}^{d}\right)$ we cannot directly apply Theorem 5.3 (b).

For $i=d\left(2^{m}+k-1\right)+j, m \in\{0,1, \ldots\}, k \in\left\{1, \ldots, 2^{m}\right\}, j \in\{1, \ldots, d\}$, let $g_{i}^{(\alpha)}, \alpha \in(0,1)$, be an element of $C_{0}^{\infty}\left([0,1] ; \mathbb{R}^{d}\right)$ such that $g_{i}^{(\alpha)}(s) \cdot e_{j^{\prime}}=0, s \in[0,1]$, for $\{1, \ldots, d\} \ni j^{\prime} \neq j$. Furthermore, 
define the $\mathbb{R}^{d}$-valued signed measures $\mu_{i}^{(\alpha)}$ and $\mu$ on $\left([0,1], \mathcal{B}([0,1])\right.$ by $\mu_{i}^{(\alpha)}((a, b]):=g_{i}^{(\alpha)}(b)-g_{i}^{(\alpha)}(a)$ and $\mu_{i}((a, b]):=g_{i}(b)-g_{i}(a)$. Suppose that $\mu_{i}^{(\alpha)}$ converges to $\mu_{i}$ in the weak*-topology as $\alpha \rightarrow 0$ and that $\left\|\mu_{i}^{(\alpha)}\right\|_{v} \leq\left\|\mu_{i}\right\|_{v}, \alpha \in(0,1)$, where $\|\cdot\|_{v}$ denotes the total variation.

Now let us study cylindrical functions $F^{(\alpha)}$ of type $F^{(\alpha)}(s, \gamma)=f\left(s ;\left\langle S_{1}^{(\alpha)}, \gamma_{s}\right\rangle, \ldots,\left\langle S_{k}^{(\alpha)}, \gamma_{s}\right\rangle\right)$ where $f, s$, and $\gamma$ are as above and $S_{i}^{(\alpha)}(s):=\int_{0}^{s} g_{i}^{(\alpha)}(u) d u$ as well as $\left\langle S_{i}^{(\alpha)}, \gamma\right\rangle:=\int_{0}^{1} g_{i}^{(\alpha)}(u) d \gamma_{u}$.

It follows that

$$
\begin{aligned}
D F^{(\alpha)}(s, \gamma)(h)=\sum_{i=1}^{k} \frac{\partial}{\partial x_{i}} f\left(s ;\left\langle S_{1}^{(\alpha)}, \gamma\right\rangle, \ldots,\left\langle S_{k}^{(\alpha)}, \gamma\right\rangle\right)\left\langle S_{i}^{(\alpha)}, h\right\rangle \\
=\sum_{i=1}^{k} \frac{\partial}{\partial x_{i}} f\left(s ;\left\langle S_{1}^{(\alpha)}, \gamma\right\rangle, \ldots,\left\langle S_{k}^{(\alpha)}, \gamma\right\rangle\right) \int_{0}^{1}\left(-g_{i}^{(\alpha)}\right)^{\prime} \cdot h d t \\
={ }_{B^{*}}\left\langle D F^{(\alpha)}(s, \gamma), h\right\rangle_{B}
\end{aligned}
$$

where this chain of equations is true for all $h \in\left\{\tilde{h}: \tilde{h} \in C\left([0,1] ; \mathbb{R}^{d}\right), h(0)=h(1)=0\right\}$. By the welldefiniteness of the second line for all $h \in L^{1}\left([0,1] ; \mathbb{R}^{d}\right)$ we may continuously extend the left-hand side as well as the right-hand side in $L^{1}\left([0,1] ; \mathbb{R}^{d}\right)$ to all $h \in L^{1}\left([0,1] ; \mathbb{R}^{d}\right)$. Furthermore, for $\Phi \in L^{1}\left([0,1]^{2} ; \mathbb{R}^{d^{2}}\right)$ introduce

$$
\left\langle\left\langle\left(S_{i}^{(\alpha)}(\cdot)\right) \otimes\left(S_{i^{\prime}}^{(\alpha)}(\cdot)\right), \Phi\right\rangle\right\rangle:=\int_{[0,1]^{2}} g_{i}^{(\alpha)}(u) \otimes g_{i^{\prime}}^{(\alpha)}(v) \cdot d \Phi(u, v) .
$$

In the same sense as above we have

$$
\begin{aligned}
& D^{2} F^{(\alpha)}(s, \gamma)(H)=\sum_{i, i^{\prime}=1}^{k} \frac{\partial^{2}}{\partial x_{i} x_{i^{\prime}}} f\left(s ;\left\langle S_{1}^{(\alpha)}, \gamma\right\rangle, \ldots,\left\langle S_{k}^{(\alpha)}, \gamma\right\rangle\right)\left\langle\left\langle\left(S_{i}^{(\alpha)}(\cdot)\right) \otimes\left(S_{i^{\prime}}^{(\alpha)}(\cdot)\right), i \circ H\right\rangle\right\rangle \\
& =\sum_{i, i^{\prime}=1}^{k} \frac{\partial^{2}}{\partial x_{i} x_{i^{\prime}}} f\left(s ;\left\langle S_{1}^{(\alpha)}, \gamma\right\rangle, \ldots,\left\langle S_{k}^{(\alpha)}, \gamma\right\rangle\right) \int_{[0,1]^{2}} g_{i}^{(\alpha)}(u) \otimes g_{i^{\prime}}^{(\alpha)}(v) \cdot d(i \circ H)(u, v) \\
& =\sum_{i, i^{\prime}=1}^{k} \frac{\partial^{2}}{\partial x_{i} x_{i^{\prime}}} f\left(s ;\left\langle S_{1}^{(\alpha)}, \gamma\right\rangle, \ldots,\left\langle S_{k}^{(\alpha)}, \gamma\right\rangle\right) \int_{[0,1]^{2}}\left(g_{i}^{(\alpha)}(u)\right)^{\prime} \otimes\left(g_{i^{\prime}}^{(\alpha)}(v)\right)^{\prime} \cdot(i \circ H)(u, v) d u d v \\
& ={ }_{\left(B \widehat{\otimes}_{\pi} B\right)^{*}}\left\langle D^{2} F^{(\alpha)}(s, \gamma), H\right\rangle_{\left(B \widehat{\otimes}_{\pi} B\right)^{* *}}, \quad H \in L^{1}\left([0,1] ; \mathbb{R}^{d}\right) \widehat{\otimes}_{\pi} L^{1}\left([0,1] ; \mathbb{R}^{d}\right) .
\end{aligned}
$$

For the next lemma recall the decomposition $X_{t}=Y_{t}+Z_{t}+A_{t}, t \geq 0$, introduced in Section 3 ,

Lemma 5.4. Suppose (3.9) and (3.10). Let $i \in \mathbb{N}$ and $T>0$.

(a) For the quadratic variation $\left[\left\langle S_{i}^{(\alpha)}, Y .\right\rangle\right]_{t}$ of $\left\langle S_{i}^{(\alpha)}, Y_{t}\right\rangle$ at $t>0$ it holds that

$$
\sup _{\alpha \in(0,1)} E\left[\left[\left\langle S_{i}^{(\alpha)}, Y .\right\rangle\right]_{t}\right]<\infty \text {. }
$$

(b) For the total variation $\left|\left\langle S_{i}^{(\alpha)}, Z .+A .\right\rangle\right|_{t}$ of $\left\langle S_{i}^{(\alpha)}, Z_{t}+A_{t}\right\rangle$ at $t>0$ it holds that

$$
\sup _{\alpha \in(0,1)} E\left[\left|\left\langle S_{i}^{(\alpha)}, Z .+A .\right\rangle\right|_{t}\right]<\infty \text {. }
$$

(c) It holds that $\left\langle S_{i}^{(\alpha)}, X_{t}\right\rangle \underset{\alpha \rightarrow 0}{\longrightarrow}\left\langle S_{i}, X_{t}\right\rangle$ uniformly on $t \in[0, T]$ almost surely. 
Proof. In the following proof we abbreviate $\|\cdot\|_{C_{0}\left([0,1] ; \mathbb{R}^{d}\right)}=\|\cdot\|_{C}$ and $\|\cdot\|_{L^{1}\left([0,1] ; \mathbb{R}^{d}\right)}=\|\cdot\|_{L^{1}}$.

(a) Using the partition of $[0, T]$ introduced in Section 3, we have

$$
\begin{aligned}
& \sup _{\alpha \in(0,1)} E\left[\left[\left\langle S_{i}^{(\alpha)}, Y .\right\rangle\right]_{T}\right]=\sup _{\alpha \in(0,1)} E\left[\lim _{n \rightarrow \infty} \sum_{j: t_{j} \leq T}\left\langle S_{i}^{(\alpha)}, Y_{t_{j}}-Y_{t_{j-1}}\right\rangle^{2}\right] \\
& =\sup _{\alpha \in(0,1)} E\left[\lim _{n \rightarrow \infty} \sum_{j: t_{j} \leq T}\left(\int_{0}^{1}\left(-g_{i}^{(\alpha)}\right)^{\prime}(u) \cdot\left(Y_{t_{j}}-Y_{t_{j-1}}\right)(u) d u\right)^{2}\right] \\
& \leq \sup _{\alpha \in(0,1)} E\left[\lim _{n \rightarrow \infty} \sum_{j: t_{j} \leq T}\left\|\left(g_{i}^{(\alpha)}\right)^{\prime}(u)\right\|_{L^{1}}^{2} \cdot\left\|Y_{t_{j}}-Y_{t_{j-1}}\right\|_{C}^{2}\right] \\
& \leq \sup _{\alpha \in(0,1)}\left\|\left(g_{i}^{(\alpha)}\right)^{\prime}(u)\right\|_{L^{1}}^{2} \cdot E\left[\lim _{n \rightarrow \infty} \sum_{j: t_{j} \leq T}\left\|Y_{t_{j}}-Y_{t_{j-1}}\right\|_{C}^{2}\right] .
\end{aligned}
$$

This proves part (a) of the lemma since $\left\|\left(g_{i}^{(\alpha)}\right)^{\prime}(u)\right\|_{L^{1}}=\left\|\mu_{i}^{(\alpha)}\right\|_{v} \leq\left\|\mu_{i}\right\|_{v}, \alpha \in(0,1)$, by hypothesis. Furthermore the second term is finite by Lemma 3.1 (a).

(b) We have

$$
\begin{aligned}
& \sup _{\alpha \in(0,1)} E\left[\left|\left\langle S_{i}^{(\alpha)}, Z .+A .\right\rangle\right|_{t}\right] \leq \sup _{\alpha \in(0,1)} E\left[\left|-\sum_{j=1}^{\infty} \int_{u=0}^{\cdot} \lambda_{j} e^{-\lambda_{j} u} W_{j}\left(e^{2 \lambda_{j} u}-1\right) d u \cdot\left\langle S_{i}^{(\alpha)}, S_{j}\right\rangle\right|_{t}\right] \\
& \left.+\sup _{\alpha \in(0,1)} E\left[\left|\sum_{j=1}^{\infty} e^{-\lambda_{j} \cdot} G_{j}(0) \cdot\left\langle S_{i}^{(\alpha)}, S_{j}\right\rangle\right|\right]_{t}\right] \\
& \leq \sum_{j=1}^{\infty} \sup _{\alpha \in(0,1)}\left\langle S_{i}^{(\alpha)}, S_{j}\right\rangle \cdot E\left[\left|\int_{u=0} \lambda_{j} e^{-\lambda_{j} u} W_{j}\left(e^{2 \lambda_{j} u}-1\right) d u\right|_{t}\right]+\sum_{j=1}^{\infty} \sup _{\alpha \in(0,1)}\left\langle S_{i}^{(\alpha)}, S_{j}\right\rangle \cdot\left|G_{j}(0)\right| \\
& =\sum_{j=1}^{\infty} \sup _{\alpha \in(0,1)}\left\langle S_{i}^{(\alpha)}, S_{j}\right\rangle \cdot\left(E\left[\int_{u=0}^{t} \lambda_{j} e^{-\lambda_{j} u}\left|W_{j}\left(e^{2 \lambda_{j} u}-1\right)\right| d u\right]+\left|G_{j}(0)\right|\right) \\
& \leq \sum_{j=1}^{\infty} \sup _{\alpha \in(0,1)}\left\langle S_{i}^{(\alpha)}, S_{j}\right\rangle \cdot\left(c t \lambda_{j}+\left|G_{j}(0)\right|\right) \\
& =\sum_{j=1}^{\infty} \sup _{\alpha \in(0,1)} \int_{0}^{1}\left(-g_{i}^{(\alpha)}\right)^{\prime}(u) \cdot S_{j}(u) d u \cdot\left(c t \lambda_{j}+\left|G_{j}(0)\right|\right) \\
& \leq \sup _{\alpha \in(0,1)}\left\|\left(g_{i}^{(\alpha)}\right)^{\prime}(u)\right\|_{L^{1}} \cdot\left\|\sum_{j=1}^{\infty}\left(c t \lambda_{j}+\left|G_{j}(0)\right|\right) \cdot S_{j}\right\|_{C}
\end{aligned}
$$

for some $c>0$ independent of $j$ and $t$. The claim now follows from

$$
\left\|\sum_{j=2}^{\infty}\left(c t \lambda_{j}+\left|G_{j}(0)\right|\right) \cdot S_{j}\right\|_{C} \leq(c T \vee 1) \sum_{m=0}^{\infty}\left(\lambda_{d 2^{m+1}}+\max _{d 2^{m}<j \leq d 2^{m+1}}\left|G_{j}(0)\right|\right)\left\|\sum_{j=d 2^{m}+1}^{d 2^{m+1}} S_{j}\right\|_{C}
$$

which is finite by $\left\|\sum_{j=d 2^{m}+1}^{d 2^{m+1}} S_{j}\right\|_{C}=2^{-\frac{m}{2}}$, (3.9), and (3.10).

(c) Let $i \in \mathbb{N}$. Furthermore let $\varepsilon>0$ and $n \in \mathbb{N}$ such that

$$
\sum_{j=n+1}^{\infty}\left|G_{j}\left(\lambda_{j} t\right)\right| \cdot\left\|S_{j}\right\|_{C}<\frac{\varepsilon}{2\left\|\mu_{i}\right\|_{v}}
$$


for all $t \in[0, T]$. For the existence of such a random $n \in \mathbb{N}$ see (2.6). We have

$$
\begin{aligned}
& \sum_{j=n+1}^{\infty} G_{j}\left(\lambda_{j} t\right)\left(\left\langle S_{i}^{(\alpha)}, S_{j}\right\rangle-\left\langle S_{i}, S_{j}\right\rangle\right)=\sum_{j=n+1}^{\infty} G_{j}\left(\lambda_{j} t\right)\left(-\int_{0}^{1} S_{j} d \mu_{i}^{(\alpha)}+\int_{0}^{1} S_{j} d \mu_{i}\right) \\
& \leq \sum_{j=n+1}^{\infty}\left|G_{j}\left(\lambda_{j} t\right)\right| \cdot\left\|S_{j}\right\|_{C} \cdot\left(\left\|\mu_{i}^{(\alpha)}\right\|_{v}+\left\|\mu_{i}\right\|_{v}\right) \leq 2 \sum_{j=n+1}^{\infty}\left|G_{j}\left(\lambda_{j} t\right)\right| \cdot\left\|S_{j}\right\|_{C} \cdot\left\|\mu_{i}\right\|_{v}<\varepsilon
\end{aligned}
$$

the second last inequality because of $\left\|\mu_{i}^{(\alpha)}\right\|_{v} \leq\left\|\mu_{i}\right\|_{v}, \alpha \in(0,1)$. Furthermore, since $\mu_{i}^{(\alpha)}$ converges to $\mu_{i}$ in the weak*-topology as $\alpha \rightarrow 0$,

$$
\begin{aligned}
& \left|\left\langle S_{i}^{(\alpha)}, X_{t}\right\rangle-\left\langle S_{i}, X_{t}\right\rangle\right| \leq \varepsilon+\left|\sum_{j=1}^{n} G_{j}\left(\lambda_{j} t\right) \cdot\left(\left\langle S_{i}^{(\alpha)}, S_{j}\right\rangle-\left\langle S_{i}, S_{j}\right\rangle\right)\right| \\
& \leq \varepsilon+\sum_{j=1}^{n}\left|G_{j}\left(\lambda_{j} t\right)\right| \cdot\left|-\int_{0}^{1} S_{j} d \mu_{i}^{(\alpha)}-\delta_{i j}\right| \\
& \underset{\alpha \rightarrow 0}{\longrightarrow} \varepsilon+\sum_{j=1}^{n}\left|G_{j}\left(\lambda_{j} t\right)\right| \cdot\left|-\int_{0}^{1} S_{j} d \mu_{i}-\delta_{i j}\right|=\varepsilon, \quad i \in \mathbb{N} .
\end{aligned}
$$

The claim follows.

Proposition 5.5. For $X_{t}$ given by (2.3) and $F\left(s, X_{s}\right)=f\left(s ;\left\langle S_{1}, X_{s}\right\rangle, \ldots,\left\langle S_{k}, X_{s}\right\rangle\right), f \in C_{0}^{\infty}\left(\mathbb{R}^{k+1}\right)$, $s \geq 0$, the following Itô formula holds.

$$
\begin{aligned}
F\left(t, X_{t}\right)= & F\left(0, X_{0}\right)+\int_{0}^{t} \frac{\partial}{\partial s} F\left(s, X_{s}\right) d s+\sum_{i=1}^{k} \int_{0}^{t} \frac{\partial}{\partial x_{i}} f\left(s ; G_{1}\left(\lambda_{1} s\right), \ldots, G_{k}\left(\lambda_{k} s\right)\right) d_{s} G_{i}\left(\lambda_{i} s\right) \\
& +\sum_{i=1}^{k} \int_{0}^{t} \lambda_{i} \frac{\partial^{2}}{\partial x_{i}^{2}} f\left(s ; G_{1}\left(\lambda_{1} s\right), \ldots, G_{k}\left(\lambda_{k} s\right)\right) d s .
\end{aligned}
$$

Proof. Next we apply the results of 14 Section 2. Let $Y_{n}, n \in \mathbb{N}$, be a sequence of $\mathbb{R}$-valued semimartingales admitting a decomposition $Y_{n}=M_{n}+V_{n}$ such that for each $t \geq 0$ it holds that $\sup _{s<t} \mid Y_{n}(s)-$ $\eta(s) \mid \underset{n \rightarrow \infty}{\longrightarrow} 0$ in probability, $\sup _{n} E\left[M_{n}(t)^{2}\right]=\sup _{n} E\left[\left[M_{n}\right]_{t}\right]<\infty$, as well as $\sup _{n} E\left[\left|V_{n}\right|_{t}\right]<\infty$. Under these conditions if $\sup _{s \leq t}\left|X_{n}(s)-\xi(s)\right| \underset{n \rightarrow \infty}{\longrightarrow} 0$ in probability then for each $T>0$

$$
\sup _{t \leq T}\left|\int_{0}^{t} X_{n}(s) d Y_{n}(s)-\int_{0}^{t} \xi(s) d \eta(s)\right| \quad \underset{n \rightarrow \infty}{\longrightarrow} 0
$$

in probability. As already mentioned, the above conclusions follow from 14 Section 2. From (5.1) it follows that

$$
\begin{aligned}
\int_{0}^{t} & B^{*}\left\langle D F^{(\alpha)}\left(s, X_{s}\right), d X_{s}\right\rangle_{B}=\lim _{\varepsilon \rightarrow 0} \int_{0}^{t}\left\langle B^{*}\left\langle F^{(\alpha)}\left(s, X_{s}\right), \frac{X_{s+\varepsilon}-X_{s}}{\varepsilon}\right\rangle_{B} d s\right. \\
= & \sum_{i=1}^{k} \lim _{\varepsilon \rightarrow 0} \int_{0}^{t} \frac{\partial}{\partial x_{i}} f\left(s ;\left\langle S_{1}^{(\alpha)}, X_{s}\right\rangle, \ldots,\left\langle S_{k}^{(\alpha)}, X_{s}\right\rangle\right) \cdot\left(\frac{\left\langle S_{i}^{(\alpha)}, X_{s+\varepsilon}\right\rangle-\left\langle S_{i}^{(\alpha)}, X_{s}\right\rangle}{\varepsilon}\right) d s \\
= & \sum_{i=1}^{k} \int_{0}^{t} \frac{\partial}{\partial x_{i}} f\left(s ;\left\langle S_{1}^{(\alpha)}, X_{s}\right\rangle, \ldots,\left\langle S_{k}^{(\alpha)}, X_{s}\right\rangle\right) \cdot d\left\langle S_{i}^{(\alpha)}, X_{s}\right\rangle
\end{aligned}
$$

where the right hand side is an Itô integral since $\left\langle S_{i}^{(\alpha)}, X_{s}\right\rangle=\sum_{j=1}^{\infty}\left\langle S_{i}^{(\alpha)}, S_{j}\right\rangle G_{j}\left(\lambda_{j} s\right)$ is a semimartingale, see Proposition 6 of 22 . From Lemma 5.4 it follows that the right-hand side converges in the sense of (5.3) to

$$
\sum_{i=1}^{k} \int_{0}^{t} \frac{\partial}{\partial x_{i}} f\left(s ;\left\langle S_{1}, X_{s}\right\rangle, \ldots,\left\langle S_{k}, X_{s}\right\rangle\right) \cdot d\left\langle S_{i}, X_{s}\right\rangle \quad \text { as } \alpha \rightarrow 0 .
$$


We recall that

$$
\Theta=2 E\left[\left(\sum_{i=1}^{\infty} \lambda_{i}^{\frac{1}{2}} \xi_{i} S_{i}\right) \otimes\left(\sum_{i^{\prime}=1}^{\infty} \lambda_{i^{\prime}}^{\frac{1}{2}} \xi_{i^{\prime}} S_{i^{\prime}}\right)\right]
$$

where $\xi_{i}, i \in \mathbb{N}$, are independent standard normal random variables. Using [12], Theorem 10.2, together with (3.10) we obtain from (5.2)

$$
\begin{aligned}
\frac{1}{2} \int_{0}^{t} & \left(B \widehat{\otimes}_{\pi} B\right)^{*} \\
= & \frac{1}{2} \sum_{l, l^{\prime}=1}^{k} \int_{0}^{t} \frac{\partial^{2}}{\partial x_{l} x_{l^{\prime}}} f\left(s ;\left\langle S_{1}^{(\alpha)}, X_{s}\right\rangle, \ldots,\left\langle S_{k}^{(\alpha)}, X_{s}\right\rangle\right) \\
& \times \int_{[0,1]^{2}}\left(g_{l}^{(\alpha)}(u)\right)^{\prime} \otimes\left(g_{l^{\prime}}^{(\alpha)}(v)\right)^{\prime} \cdot(i \circ \Theta)(u, v) d u d v d s \\
= & \sum_{l, l^{\prime}=1}^{k}\left(\lambda_{l} \lambda_{l^{\prime}}\right)^{\frac{1}{2}} \int_{0}^{t} \frac{\partial^{2}}{\partial x_{l} x_{l^{\prime}}} f\left(s ;\left\langle S_{1}^{(\alpha)}, X_{s}\right\rangle, \ldots,\left\langle S_{k}^{(\alpha)}, X_{s}\right\rangle\right) \\
& \times \int_{[0,1]^{2}}\left(g_{l}^{(\alpha)}(u)\right)^{\prime} \otimes\left(g_{l^{\prime}}^{(\alpha)}(v)\right)^{\prime} \cdot \sum_{i=1}^{\infty} S_{i}(u) \otimes S_{i}(v) d u d v d s .
\end{aligned}
$$

Here the right-hand side converges almost surely to

$$
\begin{aligned}
& \sum_{l, l^{\prime}=1}^{k}\left(\lambda_{l} \lambda_{l^{\prime}}\right)^{\frac{1}{2}} \int_{0}^{t} \frac{\partial^{2}}{\partial x_{l} x_{l^{\prime}}} f\left(s ;\left\langle S_{1}, X_{s}\right\rangle, \ldots,\left\langle S_{k}, X_{s}\right\rangle\right) \\
& \quad \times \int_{[0,1]^{2}} \sum_{i=1}^{\infty} S_{i}(u) \otimes S_{i}(v) \cdot d\left(\mu_{l}(u) \otimes \mu_{l^{\prime}}(v)\right) d s \\
& =\sum_{i=1}^{k} \lambda_{i} \int_{0}^{t} \frac{\partial^{2}}{\partial x_{i}^{2}} f\left(s ;\left\langle S_{1}, X_{s}\right\rangle, \ldots,\left\langle S_{k}, X_{s}\right\rangle\right) d s \quad \text { as } \alpha \rightarrow 0
\end{aligned}
$$

by Lemma 5.4 (c) and the hypothesis that $\mu_{i}^{(\alpha)}$ converges to $\mu_{i}$ in the weak ${ }^{*}$-topology. The proposition now follows from the Itô formula of Theorem 5.3 for $F^{(\alpha)}(s, \gamma)=f\left(s ;\left\langle S_{1}^{(\alpha)}, \gamma_{s}\right\rangle, \ldots,\left\langle S_{k}^{(\alpha)}, \gamma_{s}\right\rangle\right)$ and (5.4)-(5.7).

Remark 5.6. Reviewing the finite dimensional approximation of $X$ in Section 2 this particular specification of the Itô formula provides a certain double-check of the correctness of Proposition 4.1 and Theorem 5.3 (b).

\section{A Appendix: Some lemmas in extreme value theory}

Let $G_{i}, i \in \mathbb{N}$, be a sequence of one-dimensional Ornstein-Uhlenbeck processes of the form

$$
G_{i}(t)=G_{i}(0) e^{-t}+e^{-t} W_{i}\left(e^{2 t}-1\right), \quad t \geq 0,
$$

with independent one-dimensional standard Wiener processes $W_{i}$. Let $1 \leq \lambda_{1} \leq \lambda_{2} \leq \ldots$ be a sequence of real constants. For $T>0$ denote $G_{m, T}^{*}:=\max \left\{G_{i}\left(\lambda_{i} t\right)-G_{i}(0) e^{-\lambda_{i} t}: t \in[0, T], d 2^{m}<i \leq d 2^{m+1}\right\}$, $m \in \mathbb{Z}_{+}$. We are interested in certain moments of $G_{m, T}^{*}$. In order to obtain these moments let us first consider one Ornstein-Uhlenbeck process $G$ and $\lambda \geq 1$. Below we will use the notation $G_{T}^{*}:=$ $\max _{t \in[0, T]}\left(G(\lambda t)-G(0) e^{-\lambda t}\right)$.

Lemma A.1. The cumulative distribution function $F_{G}(x):=P\left(G_{T}^{*} \leq x\right), x \in \mathbb{R}$, is tail-equivalent to a von Mises function $F$ with $\lim _{x \rightarrow \infty}\left(1-F_{G}(x)\right) /(1-F(x))=1$. 
Proof. Let us use the representation $G(\lambda t)-G(0) e^{-\lambda t}=e^{-\lambda t} W\left(e^{2 \lambda t}-1\right), t \in[0, T]$, where $W$ is a suitable one-dimensional standard Wiener process. We have

$$
\begin{aligned}
P\left(G_{T}^{*} \leq x\right) & =P\left(\left\{G(t)-G(0) e^{-\lambda t} \leq x \text { for all } t \in[0, T]\right\}\right) \\
& =P\left(\left\{W(s) \leq x(s+1)^{\frac{1}{2}} \text { for all } s \in[0, S]\right\}\right),
\end{aligned}
$$

where $S \equiv S(\lambda)=e^{2 \lambda T}-1$. Let $\varphi$ and $\Phi$ denote the density and cumulative distribution function of the $N(0,1)$-distribution. Also let $\bar{\Phi}=1-\Phi$. Using the Corollary to Lemma 11 of $[\underline{5}$ we obtain

$$
\begin{aligned}
& P\left(G_{T}^{*}>x\right)=P\left(\left\{W(s)>x(s+1)^{\frac{1}{2}} \text { for some } s \in[0, S]\right\}\right) \\
& \quad \sim \int_{0}^{S} \frac{1}{2 s} \cdot \frac{x(s+1)^{\frac{1}{2}}}{s^{\frac{1}{2}}} \cdot \varphi\left(\frac{x(s+1)^{\frac{1}{2}}}{s^{\frac{1}{2}}}\right) d s+\bar{\Phi}\left(\frac{x(S+1)^{\frac{1}{2}}}{S^{\frac{1}{2}}}\right)=: \bar{F}(x) \equiv 1-F(x) \quad \text { as } x \rightarrow \infty
\end{aligned}
$$

in the sense that the ratio of the left-hand side of $\sim$ and the right-hand side of $\sim$ tends to one as $x \rightarrow \infty$. We also mention that in (A.1) the variable $x$ plays the role of $n$ in the Corollary to Lemma 11 of [5] and that the conditions of this corollary follow immediately.

As shown in [9], Proposition 3.3.28 and Example 3.3.23, it is now sufficient to verify

$$
\lim _{x \rightarrow \infty} \frac{(1-F(x)) F^{\prime \prime}(x)}{\left(F^{\prime}(x)\right)^{2}}=-1
$$

and $F^{\prime \prime}(x)<0$ for sufficiently large $x$. We note that

$$
\bar{\Phi}\left(\frac{x(S+1)^{\frac{1}{2}}}{S^{\frac{1}{2}}}\right)=\int_{0}^{S}\left(\frac{1}{2 s} \cdot \frac{x(s+1)^{\frac{1}{2}}}{s^{\frac{1}{2}}}-\frac{x}{2 s^{\frac{1}{2}}(s+1)^{\frac{1}{2}}}\right) \cdot \varphi\left(\frac{x(s+1)^{\frac{1}{2}}}{s^{\frac{1}{2}}}\right) d s
$$

which gives with A.1

$$
\bar{F}(x)=\int_{0}^{S} \frac{1}{s} \cdot \frac{x(s+1)^{\frac{1}{2}}}{s^{\frac{1}{2}}} \cdot \varphi\left(\frac{x(s+1)^{\frac{1}{2}}}{s^{\frac{1}{2}}}\right) d s-\int_{0}^{S} \frac{x}{2 s^{\frac{1}{2}}(s+1)^{\frac{1}{2}}} \cdot \varphi\left(\frac{x(s+1)^{\frac{1}{2}}}{s^{\frac{1}{2}}}\right) d s .
$$

We observe that with $\psi(s, x):=\varphi\left(x(s+1)^{\frac{1}{2}} \cdot s^{-\frac{1}{2}}\right)$ and $\mu(d s):=\left((s+2) \cdot(2 s)^{-\frac{1}{2}}(s+1)^{-\frac{3}{2}}\right) d s$ we have

$$
\bar{F}(x)=-\int_{0}^{S} \frac{d}{d x}\left[\varphi\left(\frac{x(s+1)^{\frac{1}{2}}}{s^{\frac{1}{2}}}\right)\right] \frac{(s+2) d s}{2 s^{\frac{1}{2}}(s+1)^{\frac{3}{2}}} \equiv x \cdot \int_{0}^{S} \psi(s, x) \cdot \frac{s+1}{s} \mu(d s)
$$

which implies

$$
\bar{F}^{\prime}(x)=\int_{0}^{S} \psi(s, x) \cdot \frac{s+1}{s} \mu(d s)-x^{2} \cdot \int_{0}^{S} \psi(s, x) \cdot\left(\frac{s+1}{s}\right)^{2} \mu(d s)
$$

and

$$
\bar{F}^{\prime \prime}(x)=-3 x \cdot \int_{0}^{S} \psi(s, x) \cdot\left(\frac{s+1}{s}\right)^{2} \mu(d s)+x^{3} \cdot \int_{0}^{S} \psi(s, x) \cdot\left(\frac{s+1}{s}\right)^{3} \mu(d s) .
$$

Since, for $k, l \in 0,1,2$,

$$
\int_{0}^{S} \psi(s, x) \cdot\left(\frac{s+1}{s}\right)^{k} \mu(d s) / \int_{0}^{S} \psi(s, x) \cdot\left(\frac{s+1}{s}\right)^{l} \mu(d s) \underset{x \rightarrow \infty}{\longrightarrow}\left(\frac{S+1}{S}\right)^{k-l}
$$

we get

$$
\lim _{x \rightarrow \infty} \frac{\bar{F}(x) \bar{F}^{\prime \prime}(x)}{\left(\bar{F}^{\prime}(x)\right)^{2}}=1
$$

which is A.2. From a.5 we deduce $F^{\prime \prime}(x)<0$ for sufficiently large $x$. The statement follows. 
Lemma A.2. (a) There exist sequences $c_{n}>0$ and $d_{n} \in \mathbb{R}, n \in \mathbb{N}$, such that

$$
\lim _{n \rightarrow \infty} F_{G}^{n}\left(c_{n} x+d_{n}\right)=\lim _{n \rightarrow \infty} F^{n}\left(c_{n} x+d_{n}\right)=e^{-e^{-x}}, \quad x \in \mathbb{R},
$$

i.e. $F_{G}$ belongs to the domain of attraction of the Gumbel distribution.

(b) The sequences $c_{n}$ and $d_{n}, n \in \mathbb{N}$, can be chosen by

$$
d_{n}:=F^{-1}\left(1-\frac{1}{n}\right) \quad \text { and } \quad c_{n}:=\frac{\bar{F}\left(d_{n}\right)}{F^{\prime}\left(d_{n}\right)},
$$

where $F^{-1}$ denotes the inverse of the restriction of $F$ to $[G(0), \infty)$.

(c) There exist the limits

$$
c:=\lim _{n \rightarrow \infty} c_{n}(\ln n)^{\frac{1}{2}}>0
$$

and

$$
d:=\lim _{n \rightarrow \infty} d_{n}(\ln n)^{-\frac{1}{2}}>0 .
$$

Proof. (a) This is a consequence of Lemma A.1 and 9] Theorem 3.3.26 as well as Proposition 3.3.28.

(b) This follows from [9] Theorem 3.3.26 and 9] Example 3.3.23.

(c) Relation (A.8) is, on the one hand, derived from

$$
-\ln \bar{F}\left(d_{n}\right)=\ln n
$$

which follows from the definition of $d_{n}$. On the other hand, according to equation (A.3) and the mean value theorem we have

$$
\bar{F}(x)=-\left.\frac{d}{d x}\left[\varphi\left(\frac{x(s+1)^{\frac{1}{2}}}{s^{\frac{1}{2}}}\right)\right]\right|_{s=S_{x}} \cdot \int_{0}^{S} \frac{(s+2) d s}{2 s^{\frac{1}{2}}(s+1)^{\frac{3}{2}}} \equiv \overline{\mathcal{F}}(x ; S) \cdot C
$$

for some $S_{x} \in[0, S]$ with $\lim _{x \rightarrow \infty} S_{x}=S$. Relation (A.7) follows now from (A.3), (A.4), and (A.6).

In Lemma A.1 and Lemma $\AA .2$ we have analyzed the cumulative distribution function $F_{G}(x):=$ $P\left(G_{T}^{*} \leq x\right), x \in \mathbb{R}$. Recall that $G_{T}^{*}=\max _{t \in[0, T]}\left(G(\lambda t)-G(0) e^{-\lambda t}\right)$. The next lemma focuses on the impact of $\lambda \geq 1$ on the sequences $c_{n} \equiv c_{n}(\lambda)>0$ and $d_{n} \equiv d_{n}(\lambda) \in \mathbb{R}, n \in \mathbb{N}$, defined in Lemma A.2

Lemma A.3. We have

$$
c_{n}(\lambda) \geq c_{0}\left((\ln n)^{\frac{1}{2}}+(\ln \lambda)^{\frac{1}{2}}\right)^{-1}
$$

and

$$
d_{n}(\lambda) \leq d_{0}\left((\ln n)^{\frac{1}{2}}+(\ln \lambda)^{\frac{1}{2}}\right)
$$

for some $c_{0}>0$ and $d_{0}>0$ independent of $n \geq 2$ and $\lambda \geq 1$.

Proof. According to A.10 it holds for all $\lambda \geq 1$ that $\bar{F}(x) \equiv \overline{\mathcal{F}}(x ; S) \cdot C(\lambda)$ where

$$
C(\lambda)=\int_{0}^{S} \frac{(s+2) d s}{2 s^{\frac{1}{2}}(s+1)^{\frac{3}{2}}} \quad \text { with } S \equiv S(\lambda)=e^{2 \lambda T}-1 .
$$

From (A.9) we obtain

$$
-\ln \left(C(\lambda) \cdot \overline{\mathcal{F}}\left(d_{n} ; S\right)\right)=\ln n,
$$

and (A.10) gives

$$
\lim _{x \rightarrow \infty} \frac{\ln (\overline{\mathcal{F}}(x ; S))}{x^{2}}=-\frac{S+1}{2 S} .
$$

Taking into consideration (A.8), using (A.14) it turns out that

$$
d_{n}^{2} \leq d_{1}(\ln n+\ln C(\lambda))
$$

for some $d_{1}>0$ independent of $n \geq 2$ and $\lambda \geq 1$. Relation A.12 follows now from (A.13). Together with Lemma A.2 (b) and (A.3), (A.4), (A.6) this implies A.11). 
Now we turn to the main object of interest of this appendix, the estimates of the particular moments of $G_{m, T}^{*}:=\max \left\{G_{i}\left(\lambda_{i} t\right)-G_{i}(0) e^{-\lambda_{i} t}: t \in[0, T], d 2^{m}<i \leq d 2^{m+1}\right\}$. Below we will use the notation $M^{(n)} \equiv M^{(n)}(\lambda):=\max \left\{\left(G_{i}(\lambda t)-G_{i}(0) e^{-\lambda t}\right): t \in[0, T], 1 \leq i \leq n\right\}$. Furthermore, let $\Gamma^{(k)}(1)$ denote the $k$ th derivative of the gamma function at $x=1$.

Lemma A.4. Let $c_{n}(\lambda)>0$ and $d_{n}(\lambda) \in \mathbb{R}$ be given by Lemma A.2 (b) and the paragraph before Lemma A.3.

(a) For $k \in \mathbb{N}$ there is an $n_{0} \equiv n_{0, k} \in \mathbb{N}$ and a constant $C \equiv C_{k}>0$, both independent of $\lambda \geq 1$, such that

$$
E\left[\left(M^{(n)}(\lambda)\right)^{k}\right] \leq C\left(d_{n}(\lambda)\right)^{k}, \quad n \geq n_{0} .
$$

(b) For each $k_{0} \in N$ there is a constant $D \equiv D_{k_{0}} \in \mathbb{R}>0$ independent of $m \in\{1,2, \ldots\}$ such that for all $1 \leq k \leq k_{0}$

$$
E\left[\left(G_{m, T}^{*}\right)^{k}\right] \leq D\left(\left(\ln \lambda_{d 2^{m+1}}\right)^{\frac{k}{2}}+m^{\frac{k}{2}}\right) .
$$

Proof. (a) By (A.1) there is some finite $a \geq 1$ independent of $\lambda \geq 1$ such that

$$
1-F_{G}(x) \leq a(1-F(x)), \quad x>0,
$$

where we mention that the functions $F_{G} \equiv F_{G, \lambda}$ and $F \equiv F_{\lambda}$ depend on $\lambda$ via $S \equiv S(\lambda)=e^{2 \lambda T}-1$. Particularly the existence of this $a \geq 1$ can be derived from $F_{\lambda}(x) / F_{G, \lambda}(x) \quad \underset{x \rightarrow \infty}{\longrightarrow} 1$ for all $\lambda \geq 1$ and $F_{\lambda}(x) \underset{\lambda \rightarrow \infty}{\longrightarrow} \infty$ for all $x>0$.

From the final conclusion of the proof of Lemma 2.2 (a) in 20 we learn that, in the notation used here, $-\ln F_{G}\left(c_{n}(\lambda) x+d_{n}(\lambda)\right) \sim 1-F_{G}\left(c_{n}(\lambda) x+d_{n}(\lambda)\right)$ as $n \rightarrow \infty$ uniformly for $x>0$ and $\lambda \geq 1$, see Lemma A.3. As in the proof of Lemma 2.2 (a) in [20, for given $\varepsilon>0$, there exist now $n_{1} \in \mathbb{N}$ independent of $\lambda \geq 1$ such that for all $n \geq n_{1}, x>0$, and $\lambda \geq 1$

$$
\begin{aligned}
& 1-F_{G}^{n}\left(c_{n}(\lambda) x+d_{n}(\lambda)\right) \leq(1+\varepsilon) n\left(1-F_{G}\left(c_{n}(\lambda) x+d_{n}(\lambda)\right)\right) \\
& \quad \leq a(1+\varepsilon) n\left(1-F\left(c_{n}(\lambda) x+d_{n}(\lambda)\right)\right) .
\end{aligned}
$$

Now we follow and modify the proof of Lemma 2.2 (a) in 20] relative to a cumulative distribution function $F$ (no longer $F_{G}$ ) that satisfies (A.1). For given $\varepsilon>0$ there is $n_{2} \in \mathbb{N}$ independent of $\lambda \geq 1$ such that, now in symbols of [20, $\left|f^{\prime}(t)\right|<\varepsilon$ if $t \geq b_{n}$ for $n \geq n_{2}$. In our notation the latter would mean $\left|\left(\bar{F}_{\lambda}(x) / F_{\lambda}^{\prime}(x)\right)^{\prime}\right|<\varepsilon$ if $x \geq d_{n}(\lambda)$ for $\lambda \geq 1$ and $n \geq n_{1}$. This holds because of (A.3)-(A.6) taking into consideration that the limit (A.6) is uniform in $\lambda \geq 1$ by $S \equiv S(\lambda)=e^{2 \lambda T}-1$. Furthermore, we can reduce the degree of $(1+\varepsilon)$ by two since, in symbols of [20] but our situation, we have $1-F\left(b_{n}\right)=n^{-1}$ and $c(x)=c, x>0$. We arrive at

$$
n\left(1-F\left(c_{n}(\lambda) x+d_{n}(\lambda)\right)\right) \leq(1+\varepsilon x)^{-1 / \varepsilon} \quad x>0, \lambda \geq 1, n \geq n_{3}
$$

for some $n_{3} \in \mathbb{N}$ independent of $\lambda \geq 1$. Thus for sufficiently small $\varepsilon>0$ there is an $n_{0} \equiv n_{0, k} \in \mathbb{N}$ such that for all $k \in \mathbb{N}$ with $\int_{0}^{\infty} k x^{k-1}\left((1+\varepsilon)(1+\varepsilon x)^{-1 / \varepsilon}\right) d x<\infty$ and all $n \geq n_{0}$ the following holds. We have

$$
1-F_{G}^{n}\left(c_{n}(\lambda) x+d_{n}(\lambda)\right) \leq a(1+\varepsilon)(1+\varepsilon x)^{-1 / \varepsilon}, \quad x>0,
$$

independent of $\lambda \geq 1$. For $n \geq n_{0}$ we obtain

$$
\begin{aligned}
E & {\left[\left(M^{(n)}(\lambda)\right)^{k}\right]=\int_{0}^{\infty} k x^{k-1} P\left(M^{(n)}(\lambda)>x\right) d x } \\
& =\int_{-d_{n}(\lambda) / c_{n}(\lambda)}^{\infty} k\left(c_{n}(\lambda) x+d_{n}(\lambda)\right)^{k-1} P\left(M^{(n)}>c_{n}(\lambda) x+d_{n}(\lambda)\right) \cdot c_{n}(\lambda) d x \\
& \leq \int_{-d_{n} / c_{n}}^{0} k\left(c_{n} x+d_{n}\right)^{k-1} \cdot c_{n} d x+\int_{0}^{\infty} k\left(c_{n} x+d_{n}\right)^{k-1}\left(1-F_{G}^{n}\left(c_{n} x+d_{n}\right)\right) \cdot c_{n} d x \\
& \leq d_{n}^{k}+a \int_{0}^{\infty} k\left(c_{n} x+d_{n}\right)^{k-1}\left((1+\varepsilon)(1+\varepsilon x)^{-1 / \varepsilon}\right) \cdot c_{n} d x .
\end{aligned}
$$


Part (a) of the lemma follows.

(b) This is a consequence of Lemma A.3, and part (a) of the present lemma.

\section{References}

[1] H. Bauer. Measure and integration theory, volume 26 of De Gruyter Stud. Math. Walter de Gruyter \& Co., Berlin, 2001.

[2] P. Billingsley. Convergence of probability measures. Wiley, New York, 1999.

[3] B. Chen and M. Csörgö. A functional modulus of continuity for a wiener process. Statist. Probab. Lett., 51(3):215-223, 2001.

[4] X. Chen and B. Wu. Functional inequality on path space over a non-compact riemannian manifold. J. Funct. Anal., 266(12):6753-6779, 2014.

[5] J. Cuzick. Boundary crossing probabilities for stationary gaussian processes and brownian motion. Trans. Amer. Math. Soc., 263(2):469-492, 1981.

[6] G. Da Prato and J. Zabczyk. Stochastic equations in infinite dimensions. Cambridge University Press, Cambridge, second edition, 2014.

[7] C. Di Girolami, G. Fabbri, and F. Russo. The covariation for banach space valued processes and applications. Metrika, 77(1):51-104, 2014.

[8] C. Di Girolami and F. Russo. Generalized covariation for banach space valued processes, itô formula and applications. Osaka J. Math., 51(3):729-783, 2014.

[9] P. Embrechts, C. Klüppelberg, and T. Mikosch. Modelling extremal events. For insurance and finance. Springer, Berlin, 1997.

[10] S. Ethier and T. Kurtz. Markov processes, Characterization and convergence. Wiley, 1986.

[11] I. Gyöngy and N. V. Krylov. On stochastics equations with respect to semimartingales. ii. it formula in banach spaces. Stochastics. An International Journal of Probability and Stochastic Processes, 6(3-4):153-173, 1981.

[12] S. Janson and S. Kaijser. Higher moments of Banach space valued random variables, volume 238 of Memoirs of the American Mathematical Society. American Mathematical Society, Providence, RI, 2015.

[13] J. Karlsson and J.-U. Löbus. A class of infinite dimensional stochastic processes. Stochastics. An International Journal of Probability and Stochastic Processes, 87(3):424-457, 2015.

[14] T. Kurtz and P. Protter. Weak limit theorems for stochastic integrals and stochastic differential equations. Ann. Probab., 19(3):1035-1070, 1991.

[15] J.-U. Löbus. A class of processes on the path space over a compact riemannian manifold with unbounded diffusion. Trans. Amer. Math. Soc., 356(9):3751-3767, 2004.

[16] T. J. Lyons and T. S. Zhang. Decomposition of dirichlet processes and its application. Ann. Prob., 22(1):494-524, 1994.

[17] M. Métivier and J. Pellaumail. Stochastic integration. Probability and Mathematical Statistics. Academic Press, New York-London-Toronto, Ont., 1980.

[18] P. Mörters and Y. Peres. Brownian Motion. Cambridge University Press, 2010.

[19] P. E. Protter. Stochastic Integration and Differential Equations. Springer, Berlin, 2005.

[20] S. I. Resnick. Extreme values, regular variation and point processes. Springer, New York, 2008. 
[21] F. Russo and P. Vallois. Stochastic calculus with respect to continuous finite quadratic variation processes. Stochastics and Stochastic Reports, 70(1-2):1-40, 2000.

[22] F. Russo and P. Vallois. Elements of stochastic calculus via regularization. Lecture Notes in Math., 1899:147-185, 2007.

[23] D. W. Stroock and S. R. S. Varadhan. Multidimensional diffusion processes. Springer, Berlin, 1979.

[24] F.-Y. Wang and B. Wu. Quasi-regular dirichlet forms on riemannian path and loop spaces. Forum Math. Volume, 20(6):1085-1096, 2008. 